TRANSACTIONS OF THE

AMERICAN MATHEMATICAL SOCIETY

Volume 357, Number 1, Pages 355-383

S 0002-9947(04)03511-1

Article electronically published on May 10, 2004

\title{
MAXIMAL HOLONOMY OF INFRA-NILMANIFOLDS WITH 2-DIMENSIONAL QUATERNIONIC HEISENBERG GEOMETRY
}

\author{
KU YONG HA, JONG BUM LEE, AND KYUNG BAI LEE
}

\begin{abstract}
Let $\mathbf{H}_{4 n-1}(\mathbb{H})$ be the quaternionic Heisenberg group of real dimension $4 n-1$ and let $I_{n}$ denote the maximal order of the holonomy groups of all infra-nilmanifolds with $\mathbf{H}_{4 n-1}(\mathbb{H})$-geometry. We prove that $I_{2}=48$. As an application, by applying Kim and Parker's result, we obtain that the minimum volume of a 2-dimensional quaternionic hyperbolic manifold with $k$ cusps is at least $\frac{\sqrt{2} k}{720}$.
\end{abstract}

\section{INTRODUCTION}

The complex Heisenberg group $\mathbf{H}_{2 n-1}(\mathbb{C})$ is

$$
\mathbf{H}_{2 n-1}(\mathbb{C})=\mathbb{R} \tilde{\times} \mathbb{C}^{n-1}
$$

with group operation given by

$$
(s, \mathbf{z})\left(t, \mathbf{z}^{\prime}\right)=\left(s+t+2 \operatorname{Im}\left\{\bar{z}^{\prime}\right\}, \mathbf{z}+\mathbf{z}^{\prime}\right),
$$

where $\operatorname{Im}\left(\mathbf{z} \overline{\mathbf{z}}^{\prime}\right)$ is the imaginary part of the complex number $z_{1} \bar{z}_{1}^{\prime}+z_{2} \bar{z}_{2}^{\prime}+\cdots+$ $z_{n-1} \bar{z}_{n-1}^{\prime}$ for $\mathbf{z}=\left(z_{1}, z_{2}, \cdots, z_{n-1}\right), \mathbf{z}^{\prime}=\left(z_{1}^{\prime}, z_{2}^{\prime}, \cdots, z_{n-1}^{\prime}\right) \in \mathbb{C}^{n-1}$. Then $\mathbf{H}_{2 n-1}(\mathbb{C})$ is a simply connected 2-step nilpotent Lie group with the center $\mathcal{Z}\left(\mathbf{H}_{2 n-1}(\mathbb{C})\right)=\mathbb{R}$. Let $M$ be an infra-nilmanifold with $\mathbf{H}_{2 n-1}(\mathbb{C})$-geometry; that is, $M=\Pi \backslash \mathbf{H}_{2 n-1}(\mathbb{C})$, where $\Pi$ is a torsion free, discrete, cocompact subgroup of $\mathbf{H}_{2 n-1}(\mathbb{C}) \rtimes C$ for some compact subgroup $C$ of $\operatorname{Aut}\left(\mathbf{H}_{2 n-1}(\mathbb{C})\right.$ ). It is well known (see for example [4]) that $\Pi$ contains a cocompact lattice of $\mathbf{H}_{2 n-1}(\mathbb{C})$ with index bounded above by a universal constant $I$. That is, $I$ is the maximal order of the holonomy groups. Note that the analogue in the Euclidean case is a consequence of a theorem of Bieberbach which states that there are only finitely many flat manifolds in each dimension. It is shown in [8] that when $n=3, I=24$. As a consequence ([8, Corollary]), the minimum volume of a complex hyperbolic 3-manifold $M$ of finite volume with $k$ cusps is $k / 9$, and $k$ is at most $-24 \pi^{3} \chi(M)$.

Received by the editors March 23, 2003 and, in revised form, August 25, 2003.

2000 Mathematics Subject Classification. Primary 20H15, 20F18, 20E99, 53C55.

Key words and phrases. Almost Bieberbach group, holonomy group, quaternionic Heisenberg group, quaternionic hyperbolic manifold.

This research was supported in part by grant No. R01-1999-000-00002-0(2002) from the interdisciplinary Research program, and by grant No. R14-2002-044-01002-0(2002) from ABRL of KOSEF

This work was done while the second-named author was visiting the Department of Mathematics at the University of Oklahoma. He expresses his sincere thanks for their hospitality.

(C)2004 American Mathematical Society 
The main concern of this paper is the quaternionic Heisenberg group

$$
\begin{aligned}
\mathfrak{N}_{n} & =\mathbf{H}_{4 n-1}(\mathbb{H})=\left\{(s, \mathbf{x}) \mid s \in \operatorname{Im}(\mathbb{H}), \mathbf{x} \in \mathbb{H}^{n-1}\right\} \\
& \approx \mathbb{R}^{3} \tilde{\times} \mathbb{H}^{n-1}
\end{aligned}
$$

with group operation given by

$$
(s, \mathbf{p})(t, \mathbf{q})=\left(s+t+2 \operatorname{Im}\left\{\mathbf{q}^{*} \cdot \mathbf{p}\right\}, \mathbf{p}+\mathbf{q}\right),
$$

for the column vectors $\mathbf{p}=\left(p_{1}, p_{2}, \cdots, p_{n-1}\right)^{t}, \mathbf{q}=\left(q_{1}, q_{2}, \cdots, q_{n-1}\right)^{t} \in \mathbb{H}^{n-1}$, where $\operatorname{Im}\left\{\mathbf{q}^{*} \cdot \mathbf{p}\right\}$ is the imaginary part of the quaternion $\bar{q}_{1} p_{1}+\bar{q}_{2} p_{2}+\cdots+\bar{q}_{n-1} p_{n-1}$ seen as an element of $\mathbb{R}^{3}$. Then $\mathfrak{N}_{n}$ is a simply connected 2-step nilpotent Lie group with the center $\mathcal{Z}\left(\mathfrak{N}_{n}\right)=\mathbb{R}^{3}$.

Let $M$ be an infra-nilmanifold with $\mathfrak{N}_{n}$-geometry; that is, $M=\Pi \backslash \mathfrak{N}_{n}$, where $\Pi \subset \mathfrak{N}_{n} \rtimes C$ is a torsion free, discrete subgroup with compact quotient, where $C$ is a compact subgroup of $\operatorname{Aut}\left(\mathfrak{N}_{n}\right)$. Such a group $\Pi$ is called an almost Bieberbach group (=AB-group). Since $\Pi \cap \mathcal{Z}\left(\mathfrak{N}_{n}\right) \cong \mathbb{Z}^{3}$ is a lattice of $\mathcal{Z}\left(\mathfrak{N}_{n}\right), M$ fits $T^{3} \rightarrow M \rightarrow N$, a Seifert 3-torus "bundle" over a $4(n-1)$-dimensional flat orbifold. When there is no singular point, it is a genuine bundle over the base space $N$ which is a flat Riemannian 4(n-1)-manifold.

It is remarkable that the group of isometries of $\mathfrak{N}_{2}$ as the boundary of the Siegel domain model of $\mathrm{H}_{\mathbb{H}}^{2}$ (that is, the nilpotent factor of the Iwasawa decomposition of the isometry group $P \mathrm{Sp}(2,1)$ of $\left.\mathrm{H}_{\mathbb{H}}^{2}\right)$ contains a maximal subgroup of automorphisms of $\mathfrak{N}_{2}$ as a real nilpotent Lie group (forgetting quaternionic structure). Therefore, the group of isometries for the natural Hermitian metric is isomorphic the group of isometries for any left invariant metric on $\mathfrak{N}_{2}$.

Let $I_{n}$ be the maximal order of the holonomy groups of all infra-nilmanifolds with $\mathfrak{N}_{n}$-geometry. Then the main result of this paper is the following.

Main Theorem. $I_{2}=48$.

The number $I_{2}$ is significant in its own right, but here is another application. According to a recent work of Kim and Parker [5, Corollary 5.3], it is related to the minimum volume of quaternionic hyperbolic orbifolds. More precisely, let $M$ be an $n$-dimensional quaternionic hyperbolic orbifold with $k$ cusps. Then the volume of $M$ is

$$
\operatorname{vol}(M) \geq \frac{2^{n-1} k}{3^{n-1}(2 n+1) m \sqrt{2}}
$$

where $m$ is the maximal index of a lattice in any of the subgroups of $\pi_{1}(M)$ stabilizing a cusp. When $n=2$, it is shown in [5, Proposition 5.8] that $m=576$. We note that this result is still true for manifolds, and in this case $m=I_{n}$. Hence we have a very sharp result for manifolds; namely, the minimum volume of a 2-dimensional quaternionic hyperbolic manifold with $k$ cusps is at least $\frac{\sqrt{2} k}{15 I_{2}}=\frac{\sqrt{2} k}{720}$.

We are indebted to the referee who made many invaluable suggestions, as well as corrections to some mistakes of the initial version of the paper.

\section{The group of isometries and affine structure of $\mathbb{R}^{3} \tilde{\times} \mathbb{R}^{4}$}

The group

$$
\mathfrak{N}_{n}=\left\{(s, \mathbf{x}) \mid s \in \operatorname{Im}(\mathbb{H}), \mathbf{x} \in \mathbb{H}^{n-1}\right\}
$$

is the nilpotent part of the Iwasawa decomposition $K A N$ of $P \operatorname{Sp}(n, 1)$. It is the boundary of the Siegel domain $\mathfrak{N}_{n} \times \mathbb{R}^{+}$. The following theorem is known (see [5]). 
Theorem 2.1. With a natural Hermitian metric on $P \operatorname{Sp}(n, 1)$, the Heisenberg isometry group is $\operatorname{Isom}_{0}\left(\mathfrak{N}_{n}\right)=\mathfrak{N}_{n} \rtimes\left(\mathrm{Sp}(n-1) \times_{\mathbb{Z}_{2}} \operatorname{Sp}(1)\right)$.

For $n=2$, this is $\mathfrak{N}_{2} \rtimes\left(\operatorname{Sp}(1) \times_{\mathbb{Z}_{2}} \operatorname{Sp}(1)\right)$. We shall explain how this acts on $\mathfrak{N}_{2}$ below.

We embed $\mathbb{H}$ into $\mathfrak{g l}(4, \mathbb{R})$ as a division algebra as follows:

$$
\zeta: \mathbb{H} \longrightarrow \mathfrak{g l}(4, \mathbb{R})
$$

defined by

$$
\zeta\left(x_{1}+x_{2} \mathbf{i}+x_{3} \mathbf{j}+x_{4} \mathbf{k}\right)=\left[\begin{array}{rrrr}
x_{1} & -x_{2} & -x_{3} & -x_{4} \\
x_{2} & x_{1} & -x_{4} & x_{3} \\
x_{3} & x_{4} & x_{1} & -x_{2} \\
x_{4} & -x_{3} & x_{2} & x_{1}
\end{array}\right] .
$$

Note that if $\mathbf{q}=x_{1}+x_{2} \mathbf{i}+x_{3} \mathbf{j}+x_{4} \mathbf{k}$ corresponds to the first column, then the second, third and fourth columns correspond to $\mathbf{q} \mathbf{i}, \mathbf{q} \mathbf{j}, \mathbf{q k}$, respectively. Then $\zeta$ is an injective homomorphism (preserving addition and multiplication).

Observe that:

(1) $\zeta(\overline{\mathbf{q}})=\zeta(\mathbf{q})^{t}$, the transpose of $\zeta(\mathbf{q})$, and

(2) $\operatorname{det}(\zeta(\mathbf{q}))=|\mathbf{q}|^{4}$.

Using the map $\zeta: \mathbb{H} \longrightarrow \mathfrak{g l}(4, \mathbb{R})$, we can embed the group $\mathfrak{N}_{2}$ into the affine group $\operatorname{Aff}\left(\mathbb{R}^{7}\right)$ as follows: For $s=s_{1} \mathbf{i}+s_{2} \mathbf{j}+s_{3} \mathbf{k}, \mathbf{x}=x_{1}+x_{2} \mathbf{i}+x_{3} \mathbf{j}+x_{4} \mathbf{k}$,

$$
(s, \mathbf{x}) \longrightarrow\left[\begin{array}{ccc}
I_{3} & -2 \bar{\xi}(\mathbf{x}) & S \\
0 & I_{4} & X \\
0 & 0 & 1
\end{array}\right] \in \operatorname{Aff}\left(\mathbb{R}^{7}\right) \subset \operatorname{GL}(8, \mathbb{R}),
$$

where $I_{3}, I_{4}$ are identity matrices of size 3 and 4 respectively,

$$
S=\left[\begin{array}{l}
s_{1} \\
s_{2} \\
s_{3}
\end{array}\right], \quad X=\left[\begin{array}{l}
x_{1} \\
x_{2} \\
x_{3} \\
x_{4}
\end{array}\right]
$$

and $\bar{\xi}(\mathbf{x})$ is obtained by removing the first row from $\zeta(\mathbf{x})^{t}$ (so it is a real $3 \times 4$ matrix). By abuse of notation, we shall use $s$ and $\mathbf{x}$ for $S$ and $X$.

Then the product in the affine group is

$$
\left[\begin{array}{ccc}
I_{3} & -2 \bar{\xi}(\mathbf{x}) & s \\
0 & I_{4} & \mathbf{x} \\
0 & 0 & 1
\end{array}\right]\left[\begin{array}{ccc}
I_{3} & -2 \bar{\xi}(\mathbf{y}) & t \\
0 & I_{4} & \mathbf{y} \\
0 & 0 & 1
\end{array}\right]=\left[\begin{array}{ccc}
I_{3} & -2 \bar{\xi}(\mathbf{x}+\mathbf{y}) & s+t-2 \bar{\xi}(\mathbf{x}) \mathbf{y} \\
0 & I_{4} & \mathbf{x}+\mathbf{y} \\
0 & 0 & 1
\end{array}\right]
$$

Since $\bar{\xi}(\mathbf{x}) \mathbf{y}=\operatorname{Im}(\overline{\mathbf{x}} \mathbf{y})=-\operatorname{Im}(\overline{\mathbf{y}} \mathbf{x})$,

$$
s+t-2 \bar{\xi}(\mathbf{x}) \mathbf{y}=s+t+2 \operatorname{Im}(\overline{\mathbf{y}} \mathbf{x}),
$$

and the above equality shows that our map $\mathfrak{N}_{2} \longrightarrow \operatorname{Aff}\left(\mathbb{R}^{7}\right)$ is an embedding.

Throughout the paper, $\sigma$ denotes the matrix

$$
\sigma=\left[\begin{array}{rrrr}
-1 & 0 & 0 & 0 \\
0 & 1 & 0 & 0 \\
0 & 0 & 1 & 0 \\
0 & 0 & 0 & 1
\end{array}\right]
$$

Note that $\sigma \in O(4)-\mathrm{SO}(4)$. 
The group of isometries of $\mathfrak{N}_{2}$ fixing the identity is

$$
\mathrm{Sp}(1) \times \mathrm{Sp}(1)=S^{3} \times S^{3} \subset \mathbb{H}^{*} \times \mathbb{H}^{*} \approx \mathrm{GL}(1, \mathbb{H}) \times \mathbb{H}^{*}
$$

which acts on the base space of the bundle $\mathbb{R}^{3} \rightarrow \mathfrak{N}_{2} \rightarrow \mathbb{H}$ as left and right translations:

$$
\begin{aligned}
\left(\mathrm{GL}(1, \mathbb{H}) \times \mathbb{H}^{*}\right) \times \mathbb{H} & \longrightarrow \mathbb{H}, \\
((c, \lambda), \mathbf{x}) & \mapsto c \mathbf{x} \lambda^{-1} .
\end{aligned}
$$

The action of this group on $\mathfrak{N}_{2}$ can be described as follows. The left multiplication by $c$ and right multiplication by $d=\lambda^{-1}$ (assuming the modulus of $c$ and $d$ are +1 ) correspond to conjugation by

$$
\left[\begin{array}{ccc}
I_{3} & 0 & 0 \\
0 & \zeta(c) & 0 \\
0 & 0 & 1
\end{array}\right] \text { and }\left[\begin{array}{ccc}
\widehat{D} & 0 & 0 \\
0 & D & 0 \\
0 & 0 & 1
\end{array}\right]
$$

where

$$
D=\sigma \zeta(\bar{d}) \sigma=\left[\begin{array}{rrrr}
d_{1} & -d_{2} & -d_{3} & -d_{4} \\
d_{2} & d_{1} & d_{4} & -d_{3} \\
d_{3} & -d_{4} & d_{1} & d_{2} \\
d_{4} & d_{3} & -d_{2} & d_{1}
\end{array}\right]
$$

and

$$
\widehat{D}=\left[\begin{array}{rrr}
d_{1}^{2}+d_{2}^{2}-d_{3}^{2}-d_{4}^{2} & 2\left(d_{2} d_{3}+d_{1} d_{4}\right) & -2\left(d_{1} d_{3}-d_{2} d_{4}\right) \\
2\left(d_{2} d_{3}-d_{1} d_{4}\right) & d_{1}^{2}-d_{2}^{2}+d_{3}^{2}-d_{4}^{2} & 2\left(d_{1} d_{2}+d_{3} d_{4}\right) \\
2\left(d_{1} d_{3}+d_{2} d_{4}\right) & -2\left(d_{1} d_{2}-d_{3} d_{4}\right) & d_{1}^{2}-d_{2}^{2}-d_{3}^{2}+d_{4}^{2}
\end{array}\right]
$$

[This matrix appears in [5] already.] Notice that $D \in \mathrm{SO}(4)$ and $\widehat{D} \in \mathrm{SO}(3)$. Thus,

$$
\left[\begin{array}{ccc}
I_{3} & 0 & 0 \\
0 & \zeta(c) & \mathbf{0} \\
0 & 0 & 1
\end{array}\right]\left[\begin{array}{ccc}
I & -2 \bar{\xi}(\mathbf{x}) & s \\
0 & I & \mathbf{x} \\
0 & 0 & 1
\end{array}\right]\left[\begin{array}{ccc}
I_{3} & 0 & 0 \\
0 & \zeta(c) & \mathbf{0} \\
0 & 0 & 1
\end{array}\right]^{-1}=\left[\begin{array}{ccc}
I & -2 \bar{\xi}(\mathbf{x}) \zeta(c)^{-1} & s \\
0 & I & \zeta(c) \mathbf{x} \\
0 & 0 & 1
\end{array}\right]
$$

and

$$
\left[\begin{array}{ccc}
\widehat{D} & 0 & 0 \\
0 & D & \mathbf{0} \\
0 & 0 & 1
\end{array}\right]\left[\begin{array}{ccc}
I & -2 \bar{\xi}(\mathbf{x}) & s \\
0 & I & \mathbf{x} \\
0 & 0 & 1
\end{array}\right]\left[\begin{array}{ccc}
\widehat{D} & 0 & 0 \\
0 & D & \mathbf{0} \\
0 & 0 & 1
\end{array}\right]^{-1}=\left[\begin{array}{ccc}
I & -2 \widehat{D} \bar{\xi}(\mathbf{x}) D^{-1} & s \\
0 & I & D \mathbf{x} \\
0 & 0 & 1
\end{array}\right]
$$

It is easy to see that

$$
\bar{\xi}(\mathbf{x}) \zeta(c)^{-1}=\bar{\xi}(\zeta(c) \mathbf{x}) \quad \text { and } \quad \widehat{D} \bar{\xi}(\mathbf{x}) D^{-1}=\bar{\xi}(D \mathbf{x})
$$

so that both $S^{3}$ 's in the group $(2-3)$ are automorphisms of $\mathfrak{N}_{2}$. If $\widehat{D}=I_{3}$, then $D= \pm I_{4}$. Therefore the two $S^{3}$ 's intersect at $\mathbb{Z}_{2}=\left\{ \pm I_{4}\right\}$.

\section{The group of AUtomorphisms of $\mathbb{R}^{3} \tilde{\times} \mathbb{R}^{4}$}

In this section, we shall calculate $\operatorname{Aut}\left(\mathfrak{N}_{2}\right)$, the group of automorphisms of the real Lie group $\mathfrak{N}_{2}$ (no quaternionic structure). From now on, we shall use $\mathfrak{N}_{2}=$ $\mathbb{R}^{3} \tilde{\times} \mathbb{R}^{4}$ rather than $\mathbb{R}^{3} \tilde{\times} \mathbb{H}$. Using the identification

$$
\begin{gathered}
\rho: \quad \mathbb{H} \longrightarrow \mathbb{R}^{4}, \\
\rho\left(x_{1}+x_{2} \mathbf{i}+x_{3} \mathbf{j}+x_{4} \mathbf{k}\right)=\left[x_{1}, x_{2}, x_{3}, x_{4}\right]^{t},
\end{gathered}
$$


we identify $\mathbb{R}^{3} \tilde{\times} \mathbb{H}$ with $\mathbb{R}^{3} \tilde{\times} \mathbb{R}^{4}$ by

$$
(s, \mathbf{q}) \longleftrightarrow(s, \rho(\mathbf{q})) .
$$

Accordingly, we introduce a new notation for $\operatorname{Im}\{\overline{\mathbf{y}} \mathbf{x}\}$. For

$$
\mathbf{x}=\left[\begin{array}{l}
x_{1} \\
x_{2} \\
x_{3} \\
x_{4}
\end{array}\right], \quad \mathbf{y}=\left[\begin{array}{l}
y_{1} \\
y_{2} \\
y_{3} \\
y_{4}
\end{array}\right]
$$

define

$$
\mathcal{I}(\mathbf{x}, \mathbf{y})=\left[\begin{array}{l}
-\left|\begin{array}{ll}
x_{1} & y_{1} \\
x_{2} & y_{2}
\end{array}\right|+\left|\begin{array}{ll}
x_{3} & y_{3} \\
x_{4} & y_{4}
\end{array}\right| \\
-\left|\begin{array}{ll}
x_{1} & y_{1} \\
x_{3} & y_{3}
\end{array}\right|-\left|\begin{array}{ll}
x_{2} & y_{2} \\
x_{4} & y_{4}
\end{array}\right| \\
-\left|\begin{array}{ll}
x_{1} & y_{1} \\
x_{4} & y_{4}
\end{array}\right|+\left|\begin{array}{ll}
x_{2} & y_{2} \\
x_{3} & y_{3}
\end{array}\right|
\end{array}\right] .
$$

We shall represent $\mathcal{I}(\mathbf{x}, \mathbf{y})$ by matrix products as follows. Using the function $\zeta: \mathbb{H} \longrightarrow \mathbb{R}^{4}$ in $(2-1)$, let

$$
K_{1}=\zeta(-\mathbf{i}), \quad K_{2}=\zeta(-\mathbf{j}), \quad K_{3}=\zeta(-\mathbf{k}) .
$$

Then

$$
K_{1}=\left[\begin{array}{rrrr}
0 & 1 & 0 & 0 \\
-1 & 0 & 0 & 0 \\
0 & 0 & 0 & 1 \\
0 & 0 & -1 & 0
\end{array}\right], K_{2}=\left[\begin{array}{rrrr}
0 & 0 & 1 & 0 \\
0 & 0 & 0 & -1 \\
-1 & 0 & 0 & 0 \\
0 & 1 & 0 & 0
\end{array}\right], K_{3}=\left[\begin{array}{rrrr}
0 & 0 & 0 & 1 \\
0 & 0 & 1 & 0 \\
0 & -1 & 0 & 0 \\
-1 & 0 & 0 & 0
\end{array}\right] \text {. }
$$

Let $\sigma$ be the matrix in (2-2) and

$$
J_{i}=\sigma^{-1} K_{i} \sigma, \quad i=1,2,3,
$$

so that

$$
J_{1}=\left[\begin{array}{rrrr}
0 & -1 & 0 & 0 \\
1 & 0 & 0 & 0 \\
0 & 0 & 0 & 1 \\
0 & 0 & -1 & 0
\end{array}\right], J_{2}=\left[\begin{array}{rrrr}
0 & 0 & -1 & 0 \\
0 & 0 & 0 & -1 \\
1 & 0 & 0 & 0 \\
0 & 1 & 0 & 0
\end{array}\right], J_{3}=\left[\begin{array}{rrrr}
0 & 0 & 0 & -1 \\
0 & 0 & 1 & 0 \\
0 & -1 & 0 & 0 \\
1 & 0 & 0 & 0
\end{array}\right] \text {. }
$$

Then, if $\mathbf{x}=\rho(\mathbf{q})$, we have

$$
\begin{aligned}
& J_{1} \mathbf{x}=\rho(\mathbf{q i}), \quad J_{2} \mathbf{x}=\rho(\mathbf{q} \mathbf{j}), \quad J_{3} \mathbf{x}=\rho(\mathbf{q k}), \\
& -K_{1} \mathbf{x}=\rho(\mathbf{i q}), \quad-K_{2} \mathbf{x}=\rho(\mathbf{j q}), \quad-K_{3} \mathbf{x}=\rho(\mathbf{k q}) .
\end{aligned}
$$

The definition of $\mathcal{I}$ in $3-2$ becomes

$$
\mathcal{I}(\mathbf{x}, \mathbf{y})=\left(\mathbf{x}^{t} J_{1} \mathbf{y}, \mathbf{x}^{t} J_{2} \mathbf{y}, \mathbf{x}^{t} J_{3} \mathbf{y}\right)^{t} .
$$

Clearly $\mathcal{I}(\mathbf{x}, \mathbf{y})=-\mathcal{I}(\mathbf{y}, \mathbf{x})$ and $\mathcal{I}(\mathbf{x}, \mathbf{y})$ corresponds to $\operatorname{Im}\{\overline{\mathbf{y}} \mathbf{x}\}$. Thus the group operation in $\mathfrak{N}_{2}=\mathbb{R}^{3} \tilde{\times} \mathbb{R}^{4}$ becomes

$$
(s, \mathbf{x})(t, \mathbf{y})=(s+t+2 \mathcal{I}(\mathbf{x}, \mathbf{y}), \mathbf{x}+\mathbf{y}) .
$$

Since $\mathcal{I}(\mathbf{x}, \pm \mathbf{x})=0$, we see easily that

$$
(s, \mathbf{x})^{-1}=(-s,-\mathbf{x}) .
$$


Thus we have

$$
[(s, \mathbf{x}),(t, \mathbf{y})]=(s, \mathbf{x})^{-1}(t, \mathbf{y})^{-1}(s, \mathbf{x})(t, \mathbf{y})=(4 \mathcal{I}(\mathbf{x}, \mathbf{y}), \mathbf{0}) .
$$

Clearly $J_{1}, J_{2}, J_{3}$ together with $K_{1}, K_{2}, K_{3}$ form a linear basis for the vector space $\mathfrak{s o}(4, \mathbb{R})$ of the skew-symmetric matrices. We denote the two subspaces of $\mathfrak{s o}(4, \mathbb{R})$ as follows:

$$
\begin{aligned}
& \mathfrak{s o}(4, \mathbb{R})_{\ell}=\left\langle J_{1}, J_{2}, J_{3}\right\rangle, \\
& \mathfrak{s o}(4, \mathbb{R})_{r}=\left\langle K_{1}, K_{2}, K_{3}\right\rangle .
\end{aligned}
$$

Since $J_{i} K_{j}=K_{j} J_{i}$ for all $i, j=1,2,3, \mathfrak{s o}(4, \mathbb{R})_{\ell}$ and $\mathfrak{s o}(4, \mathbb{R})_{r}$ are ideals of $\mathfrak{s o}(4, \mathbb{R})$ and

$$
\mathfrak{s o}(4, \mathbb{R})=\mathfrak{s o}(4, \mathbb{R})_{\ell} \oplus \mathfrak{s o}(4, \mathbb{R})_{r} .
$$

For any $C \in \operatorname{GL}(4, \mathbb{R})$ and $V \in \mathfrak{s o}(4, \mathbb{R})$,

$$
J_{C}(V)=C^{t} V C
$$

defines a linear isomorphism $J_{C}: \mathfrak{s o}(4, \mathbb{R}) \longrightarrow \mathfrak{s o}(4, \mathbb{R})$. In fact, with respect to the basis $\left\{J_{1}, J_{2}, J_{3}, K_{1}, K_{2}, K_{3}\right\}$, it turns out that $\operatorname{det}\left(J_{C}\right)=(\operatorname{det}(C))^{3}$.

In order to calculate the group of automorphisms of $\mathfrak{N}_{2}$, first we define

$$
O(\mathbf{J}, 4)=\left\{C \in \operatorname{GL}(4, \mathbb{R}) \mid C^{t} J_{i} C \in \mathfrak{s o}(4, \mathbb{R})_{\ell} \text { for } i=1,2,3\right\} .
$$

Then $C \in O(\mathbf{J}, 4)$ if and only if the map $J_{C}$ leaves the subspace $\mathfrak{s o}(4, \mathbb{R})_{\ell}$ spanned by $J_{1}, J_{2}, J_{3}$ invariant. Therefore,

$$
C^{t} J_{i} C=\lambda_{i 1} J_{1}+\lambda_{i 2} J_{2}+\lambda_{i 3} J_{3}, \quad \lambda_{i j} \in \mathbb{R},
$$

for $i=1,2,3$. Then it turns out that the matrix $\lambda=\left(\lambda_{i j}\right)$ is non-singular.

Now we form the column vector

$$
\mathbf{J}=\left[\begin{array}{l}
J_{1} \\
J_{2} \\
J_{3}
\end{array}\right]
$$

with entries the matrices $J_{1}, J_{2}, J_{3}$. With some abuse of notation, we can write

$$
O(\mathbf{J}, 4)=\left\{C \in \mathrm{GL}(4, \mathbb{R}) \mid C^{t} \mathbf{J} C=\lambda \mathbf{J}, \lambda \in \mathrm{GL}(3, \mathbb{R})\right\} .
$$

Clearly $O(\mathbf{J}, 4)$ is a closed subgroup of $\mathrm{GL}(4, \mathbb{R})$. For $C \in O(\mathbf{J}, 4)$, let $\widehat{C} \in \mathrm{GL}(3, \mathbb{R})$ denote the non-singular $3 \times 3$ matrix $\lambda$ which satisfies $C^{t} \mathbf{J} C=\lambda \mathbf{J}$. Therefore,

$$
C^{t} \mathbf{J} C=\widehat{C} \mathbf{J}
$$

Then $C \mapsto \widehat{C}$ defines a homomorphism $\epsilon: O(\mathbf{J}, 4) \rightarrow \mathrm{GL}(3, \mathbb{R})$ by

$$
\epsilon(C)=\widehat{C}
$$

so that $C^{t} \mathbf{J} C=\epsilon(C) \mathbf{J}$.

Lemma 3.1. (1) $\epsilon$ is a homomorphism.

(2) $C \mapsto\left(\frac{1}{\sqrt{|\operatorname{det}(C)|}} \widehat{C}, \sqrt{|\operatorname{det}(C)|}\right)$ defines a homomorphism $\widehat{:}: O(\mathbf{J}, 4) \longrightarrow$ $O(3) \times \mathbb{R}^{+}$. Moreover, $\operatorname{det}(\widehat{C})^{2}=|\operatorname{det}(C)|^{3}$.

(3) $O(\mathbf{J}, 4)$ is closed under transpose and the above homomorphism $\epsilon$ commutes with transpose. 
Proof. For $C, D \in O(\mathbf{J}, 4)$,

$$
\begin{aligned}
(C D)^{t} \mathbf{J}(C D) & =D^{t}\left(C^{t} \mathbf{J} C\right) D \\
& =D^{t}(\widehat{C} \mathbf{J}) D \\
& =\widehat{C} \cdot D^{t} \mathbf{J} D \text { (since } \widehat{C} \text { is a "scalar" matrix) } \\
& =\widehat{C} \widehat{D} \mathbf{J}
\end{aligned}
$$

shows $\epsilon(C D)=\epsilon(C) \epsilon(D)$.

Now assume that $C \in O(\mathbf{J}, 4)$ and $\operatorname{det}(C)= \pm 1$. Let $\widehat{C}=\left(\lambda_{i j}\right)$. Then the equalities (3-5) are satisfied. A direct calculation from these equalities shows that

$$
|\operatorname{det}(C)|=\lambda_{i 1}^{2}+\lambda_{i 2}^{2}+\lambda_{i 3}^{2}
$$

for all $i=1,2,3$. Let $\mathbf{c}_{i}$ be the $i$ th column of the matrix $C$. Then

$$
\mathcal{I}\left(\mathbf{c}_{i}, \mathbf{c}_{j}\right)=\left[\begin{array}{l}
\mathbf{c}_{i}{ }^{t} J_{1} \mathbf{c}_{j} \\
\mathbf{c}_{i}{ }^{t} J_{2} \mathbf{c}_{j} \\
\mathbf{c}_{i}{ }^{t} J_{3} \mathbf{c}_{j}
\end{array}\right]=\left[\begin{array}{l}
\left(C^{t} J_{1} C\right)_{(i, j)} \\
\left(C^{t} J_{2} C\right)_{(i, j)} \\
\left(C^{t} J_{3} C\right)_{(i, j)}
\end{array}\right] .
$$

From the equalities (3-5), we have

$$
\begin{aligned}
& -\left(C^{t} J_{1} C\right)_{(1,2)}=\lambda_{11}=\left(C^{t} J_{1} C\right)_{(3,4)}, \\
& -\left(C^{t} J_{2} C\right)_{(1,2)}=\lambda_{21}=\left(C^{t} J_{2} C\right)_{(3,4)}, \\
& -\left(C^{t} J_{3} C\right)_{(1,2)}=\lambda_{31}=\left(C^{t} J_{3} C\right)_{(3,4)} .
\end{aligned}
$$

Consequently, we have

$$
-\mathcal{I}\left(\mathbf{c}_{1}, \mathbf{c}_{2}\right)=\left[\begin{array}{l}
\lambda_{11} \\
\lambda_{21} \\
\lambda_{31}
\end{array}\right]=\mathcal{I}\left(\mathbf{c}_{3}, \mathbf{c}_{4}\right)
$$

Similarly, the second and third columns of $\widehat{C}=\left(\lambda_{i j}\right)$ are

$$
\begin{aligned}
& -\mathcal{I}\left(\mathbf{c}_{1}, \mathbf{c}_{3}\right)=-\mathcal{I}\left(\mathbf{c}_{2}, \mathbf{c}_{4}\right), \\
& -\mathcal{I}\left(\mathbf{c}_{1}, \mathbf{c}_{4}\right)=\mathcal{I}\left(\mathbf{c}_{2}, \mathbf{c}_{3}\right)
\end{aligned}
$$

so that

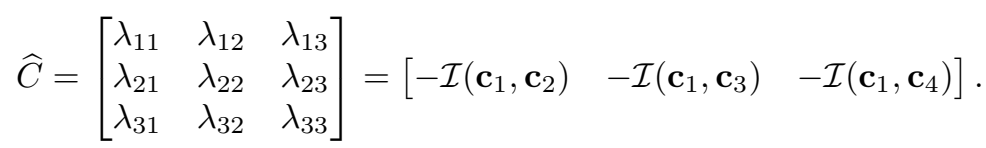

For the sake of calculation, we set the second matrix to $\left(\lambda_{i j}^{\prime}\right)$, so

$$
\widehat{C}=\left[\begin{array}{ccc}
\lambda_{11}^{\prime} & \lambda_{12}^{\prime} & \lambda_{13}^{\prime} \\
\lambda_{21}^{\prime} & \lambda_{22}^{\prime} & \lambda_{23}^{\prime} \\
\lambda_{31}^{\prime} & \lambda_{32}^{\prime} & \lambda_{33}^{\prime}
\end{array}\right]=\left[\begin{array}{lll}
\mathcal{I}\left(\mathbf{c}_{3}, \mathbf{c}_{4}\right) & -\mathcal{I}\left(\mathbf{c}_{2}, \mathbf{c}_{4}\right) & \mathcal{I}\left(\mathbf{c}_{2}, \mathbf{c}_{3}\right)
\end{array}\right]
$$

Of course, then $\lambda_{i j}=\lambda_{i j}^{\prime}$ for all $i, j$. For $j \neq k$, using the equalities (3-6) and (3-7), it can be shown that

$$
\begin{aligned}
& 2\left(\lambda_{j 1} \lambda_{k 1}+\lambda_{j 2} \lambda_{k 2}+\lambda_{j 3} \lambda_{k 3}\right) \\
= & \left(\lambda_{j 1} \lambda_{k 1}^{\prime}+\lambda_{j 2} \lambda_{k 2}^{\prime}+\lambda_{j 3} \lambda_{k 3}^{\prime}\right)+\left(\lambda_{j 1}^{\prime} \lambda_{k 1}+\lambda_{j 2}^{\prime} \lambda_{k 2}+\lambda_{j 3}^{\prime} \lambda_{k 3}\right) \\
= & 0 .
\end{aligned}
$$


Thus any two row vectors of $\widehat{C}$ are orthogonal. Consequently if $C \in O(\mathbf{J}, 4)$ with $\operatorname{det}(C)= \pm 1$, then $\widehat{C} \in O(3)$.

Suppose $C \in O(\mathbf{J}, 4)$. Let $d=\frac{1}{\sqrt[4]{|\operatorname{det}(C)|}}$ and $D=d I \in \mathrm{GL}(4, \mathbb{R})$, a diagonal matrix. Then $D \in O(\mathbf{J}, 4)$ and $d C=D C \in O(\mathbf{J}, 4)$ with $\operatorname{det}(d C)= \pm 1$. By the above argument, $\widehat{d C} \in O(3)$ and $\widehat{d C}=\widehat{D} \widehat{C}=d^{2} \widehat{C}$. Hence $\frac{1}{\sqrt{|\operatorname{det}(C)|}} \widehat{C} \in O(3)$. This shows that

$$
\text { ^: } O(\mathbf{J}, 4) \longrightarrow O(3) \times \mathbb{R}^{+}, \quad C \mapsto\left(\frac{1}{\sqrt{|\operatorname{det}(C)|}} \widehat{C}, \sqrt{|\operatorname{det}(C)|}\right)
$$

is a homomorphism and $\operatorname{det}(\widehat{C})^{2}=|\operatorname{det}(C)|^{3}$.

Next suppose $C \in O(\mathbf{J}, 4)$. Let $\widehat{C}=\left(\lambda_{i j}\right)$. From the equalities (3-5), we have $J_{1}^{2}=-I_{4}, J_{1} J_{2}=-J_{2} J_{1}$, etc. Therefore,

$$
\left(\lambda_{i 1} J_{1}+\lambda_{i 2} J_{2}+\lambda_{i 3} J_{3}\right)^{2}=-|\operatorname{det}(C)| I_{4} .
$$

Taking inverse matrices of the equalities (3-5), we get

$$
\begin{aligned}
-C^{-1} J_{i}\left(C^{-1}\right)^{t} & =\left(C^{t} J_{i} C\right)^{-1} \\
& =\left(\lambda_{i 1} J_{1}+\lambda_{i 2} J_{2}+\lambda_{i 3} J_{3}\right)^{-1} \\
& =-\frac{1}{|\operatorname{det}(C)|}\left(\lambda_{i 1} J_{1}+\lambda_{i 2} J_{2}+\lambda_{i 3} J_{3}\right) .
\end{aligned}
$$

This shows that $\left(C^{-1}\right)^{t}$ is also an element of $O(\mathbf{J}, 4)$ with

$$
\left(\left(C^{-1}\right)^{t}\right)^{\wedge}=\frac{1}{|\operatorname{det}(C)|} \widehat{C}
$$

Suppose $\operatorname{det}(C)= \pm 1$. Then, from the above equality, we have

$$
\begin{aligned}
\left(C^{t}\right)^{\wedge} & =\left(C^{-1}\right)^{\wedge} \\
& =\left(C^{\wedge}\right)^{-1}(\text { since } \epsilon \text { is a homomorphism }) \\
& =\left(C^{\wedge}\right)^{t}(\text { since } \widehat{C} \in O(3)) .
\end{aligned}
$$

One can now easily see the equality $\left(C^{t}\right)^{\wedge}=\left(C^{\wedge}\right)^{t}$ for any $C \in O(\mathbf{J}, 4)$. This proves the lemma.

Let $S^{3} \subset \mathbb{H}^{*}$ be the group of unit quaternions

$$
S^{3}=\left\{x_{1}+x_{2} \mathbf{i}+x_{3} \mathbf{j}+x_{4} \mathbf{k} \mid x_{1}^{2}+x_{2}^{2}+x_{3}^{2}+x_{4}^{2}=1\right\} .
$$

Using $\zeta: \mathbb{H} \longrightarrow \mathfrak{g l}(4, \mathbb{R})$ of $(\underline{2-1})$, we obtain two copies of $S^{3}$ in $O(\mathbf{J}, 4)$ :

$$
\begin{aligned}
& S_{\ell}^{3}=\zeta\left(S^{3}\right), \\
& S_{r}^{3}=\sigma \zeta\left(S^{3}\right) \sigma .
\end{aligned}
$$

Then

Proposition 3.2. $\quad(1) \epsilon$ has image $\epsilon\left(S_{r}^{3} \times \mathbb{R}\right)=\mathrm{SO}(3) \times \mathbb{R}^{+}$.

(2) $\epsilon$ has kernel $S_{\ell}^{3}$ so that $O(\mathbf{J}, 4)=\mathrm{SO}(4) \times \mathbb{R}^{+}=\left(S_{\ell}^{3} \times \mathbb{Z}_{2} S_{r}^{3}\right) \times \mathbb{R}^{+}$. 
Proof. For $\mathbf{q}=x_{1}+x_{2} \mathbf{i}+x_{3} \mathbf{j}+x_{4} \mathbf{k}$,

$$
\begin{aligned}
\zeta(\mathbf{q}) & =\left[\begin{array}{rrrr}
x_{1} & -x_{2} & -x_{3} & -x_{4} \\
x_{2} & x_{1} & -x_{4} & x_{3} \\
x_{3} & x_{4} & x_{1} & -x_{2} \\
x_{4} & -x_{3} & x_{2} & x_{1}
\end{array}\right] \in S_{\ell}^{3}, \\
\sigma \zeta(\overline{\mathbf{q}}) \sigma= & {\left[\begin{array}{rrrr}
x_{1} & -x_{2} & -x_{3} & -x_{4} \\
x_{2} & x_{1} & x_{4} & -x_{3} \\
x_{3} & -x_{4} & x_{1} & x_{2} \\
x_{4} & x_{3} & -x_{2} & x_{1}
\end{array}\right] \in S_{r}^{3} }
\end{aligned}
$$

with

$$
\begin{aligned}
\zeta(\mathbf{q})^{\wedge} & =I_{3}, \\
(\sigma \zeta(\overline{\mathbf{q}}) \sigma)^{\wedge} & =\left[\begin{array}{rrr}
x_{1}^{2}+x_{2}^{2}-x_{3}^{2}-x_{4}^{2} & 2\left(x_{2} x_{3}+x_{1} x_{4}\right) & -2\left(x_{1} x_{3}-x_{2} x_{4}\right) \\
2\left(x_{2} x_{3}-x_{1} x_{4}\right) & x_{1}^{2}-x_{2}^{2}+x_{3}^{2}-x_{4}^{2} & 2\left(x_{1} x_{2}+x_{3} x_{4}\right) \\
2\left(x_{1} x_{3}+x_{2} x_{4}\right) & -2\left(x_{1} x_{2}-x_{3} x_{4}\right) & x_{1}^{2}-x_{2}^{2}-x_{3}^{2}+x_{4}^{2}
\end{array}\right] .
\end{aligned}
$$

See the equalities (2-1), (2-5) and (2-6).

Then $S_{\ell}^{3} \times S_{r}^{3}$ corresponds to $S^{3} \times S^{3} \subset \mathbb{H}^{*} \times \mathbb{H}^{*}=\mathrm{GL}(1, \mathbb{H}) \times \mathbb{H}^{*}$ in (2-4) acting on $\mathbb{H}$, by left and right multiplications. Clearly, $S_{\ell}^{3} \cap S_{r}^{3}=\mathbb{Z}_{2}=\left\{ \pm I_{4}\right\}$ and

$$
S_{\ell}^{3} \times_{\mathbb{Z}_{2}} S_{r}^{3}=\mathrm{SO}(4) \subset O(\mathbf{J}, 4) .
$$

From the equalities (3-10), the map

$$
\epsilon: O(\mathbf{J}, 4) \rightarrow O(3) \times \mathbb{R}^{+}
$$

maps $S_{r}^{3}$ onto $\mathrm{SO}(3)$ (with kernel $\mathbb{Z}_{2}=\left\{ \pm I_{4}\right\}$ ) and $S_{\ell}^{3}$ trivially.

Next we observe that the matrix $\operatorname{diag}\{1,1,-1\}$ is not in the image of the homomorphism. Otherwise, there would be $C \in O(\mathbf{J}, 4)$ so that

$$
C^{t} J_{1} C=J_{1}, C^{t} J_{2} C=J_{2}, C^{t} J_{3} C=-J_{3} .
$$

Since $J_{1} J_{2}=-J_{3}$, we would have

$$
\begin{aligned}
J_{1} J_{2} & =-J_{3}=C^{t} J_{3} C=-C^{t} J_{1} J_{2} C \\
& =-C^{t} J_{1} C \cdot C^{-1}\left(C^{t}\right)^{-1} \cdot C^{t} J_{2} C \\
& =-J_{1}\left(C^{t} C\right)^{-1} J_{2},
\end{aligned}
$$

and $C^{t} C=-I$, and hence the $(1,1)$-entry would be $\sum_{i} c_{i 1}^{2}=-1$, which is impossible. Thus $\operatorname{diag}\{1,1,-1\}$ is not in the image of $\epsilon$. Now suppose there exists $C \in O(\mathbf{J}, 4)$ so that $\widehat{C} \in O(3) \backslash \operatorname{SO}(3)$. Then we can write $\widehat{C}=\operatorname{diag}\{1,1,-1\} \widehat{E}$ for some $E \in O(\mathbf{J}, 4)$. Hence $\widehat{C E^{-1}}=\operatorname{diag}\{1,1,-1\}$. But the right-hand side is not in the image of the homomorphism. This proves that

$$
\epsilon: O(\mathbf{J}, 4) \longrightarrow \mathrm{SO}(3) \times \mathbb{R}^{+}
$$

is a surjective homomorphism. (Recall, for $s \neq 0, s I_{4} \in O(\mathbf{J}, 4)$ with $(s I)^{\wedge}=s^{2} I_{3}$.)

Suppose that $C \in \operatorname{ker}(\epsilon)$. Then $\widehat{C}=I$ and thus $C^{t} J_{i} C=J_{i}$ for $i=1,2,3$. Since $J_{1} J_{2}=-J_{3}$, we have

$$
\begin{aligned}
J_{1} J_{2} & =-J_{3}=-C^{t} J_{3} C=C^{t} J_{1} J_{2} C=C^{t} J_{1} C \cdot C^{-1}\left(C^{t}\right)^{-1} \cdot C^{t} J_{2} C \\
& =J_{1}\left(C^{t} C\right)^{-1} J_{2},
\end{aligned}
$$


$C^{t} C=I$ and $C \in O(4)$. On the other hand, $C^{t} J_{1} C=J_{1}$ and $C^{t} J_{2} C=J_{2}$ immediately induce that $C$ is of the form $\zeta(\mathbf{q})$ for some $\mathbf{q} \in S_{\ell}^{3}$. Since we know that $S_{\ell}^{3}$ is already in the kernel of $\epsilon$, we conclude that

$$
\operatorname{ker}(\epsilon)=S_{\ell}^{3} .
$$

Thus we have obtained an exact sequence

$$
1 \rightarrow S_{\ell}^{3} \rightarrow O(\mathbf{J}, 4) \rightarrow \mathrm{SO}(3) \times \mathbb{R}^{+} \rightarrow 1
$$

and equalities

$$
\begin{aligned}
O(\mathbf{J}, 4) & =\left(S_{\ell}^{3} \times_{\mathbb{Z}_{2}} S_{r}^{3}\right) \times \mathbb{R}^{+} \\
& =\mathrm{SO}(4) \times \mathbb{R}^{+} .
\end{aligned}
$$

It is clear that $S_{\ell}^{3}$ and $S_{r}^{3}$ and $\mathbb{R}^{+}$commute with each other.

Corollary 3.3. If $C \in O(\mathbf{J}, 4)$, then

$$
\operatorname{det}(\widehat{C})=\operatorname{det}(C)^{3 / 2} .
$$

Proof. Follows from Lemma 3.1 and Proposition 3.2

Lemma 3.4. Every matrix in $S_{\ell}^{3} \cup S_{r}^{3}$ except $\pm I$ has eigenvalues $a+b \mathbf{i}, a+b \mathbf{i}$, $a-b \mathbf{i}, a-b \mathbf{i}$ with $b \neq 0$ (two complex double roots). Conversely, suppose $A \in \mathrm{SO}(4)$ has eigenvalues $a \pm b \mathbf{i}$ with $b \neq 0$ of multiplicity 2. Then either $A \in S_{\ell}^{3}$ or $A \in S_{r}^{3}$.

Proof. Let $A \in \mathrm{SO}(4)$ have multiple eigenvalues $a \pm b \mathbf{i}$ with $b \neq 0$. Looking at the standard maximal torus of $\mathrm{SO}(4)$, there is $P \in \mathrm{SO}(4)$ such that $P A P^{-1}=$ $\left[\begin{array}{cc}R(\theta) & 0 \\ 0 & R\left(\theta^{\prime}\right)\end{array}\right]$, where $R(\theta)=\left[\begin{array}{cc}\cos \theta & -\sin \theta \\ \sin \theta & \cos \theta\end{array}\right]$. Since $A \in \mathrm{SO}(4)$ has multiple eigenvalues $a \pm b \mathbf{i}$ with $b \neq 0$, either $\theta=\theta^{\prime}$ or $\theta=-\theta^{\prime}$. If $P A P^{-1}=\left[\begin{array}{cc}R(\theta) & 0 \\ 0 & R(\theta)\end{array}\right]$, then $P A P^{-1} \in S_{\ell}^{3}$; if $P A P^{-1}=\left[\begin{array}{cc}R(\theta) & 0 \\ 0 & R(-\theta)\end{array}\right]$, then $P A P^{-1} \in S_{r}^{3}$. Since $S_{\ell}^{3}$ and $S_{r}^{3}$ are normal subgroups in $\mathrm{SO}(4), A \in S_{r}^{3}$ or $A \in S_{\ell}^{3}$.

Since the center, $\mathcal{Z}\left(\mathfrak{N}_{2}\right)=\mathbb{R}^{3}$, is a characteristic subgroup of $\mathfrak{N}_{2}$, every automorphism of $\mathfrak{N}_{2}$ restricts to an automorphism of $\mathbb{R}^{3}$. Consequently an automorphism of $\mathfrak{N}_{2}$ induces an automorphism on the quotient group $\mathbb{R}^{4}$. Thus there is a natural homomorphism $\operatorname{Aut}\left(\mathfrak{N}_{2}\right) \rightarrow \operatorname{Aut}\left(\mathbb{R}^{3}\right) \times \operatorname{Aut}\left(\mathbb{R}^{4}\right), \theta \mapsto(\hat{\theta}, \bar{\theta})$.

Lemma 3.5. Image $\left\{\operatorname{Aut}\left(\mathfrak{N}_{2}\right) \rightarrow \operatorname{Aut}\left(\mathbb{R}^{4}\right)\right\}=O(\mathbf{J}, 4)$. Moreover, the exact sequence $\operatorname{Aut}\left(\mathfrak{N}_{2}\right) \rightarrow O(\mathbf{J}, 4) \rightarrow 1$ splits.

Proof. Let $\theta \in \operatorname{Aut}\left(\mathfrak{N}_{2}\right)$. Then $(\hat{\theta}, \bar{\theta}) \in \operatorname{Aut}\left(\mathbb{R}^{3}\right) \times \operatorname{Aut}\left(\mathbb{R}^{4}\right)$. Since $[(s, \mathbf{x}),(t, \mathbf{y})]=$ $(4 \mathcal{I}(\mathbf{x}, \mathbf{y}), \mathbf{0})$,

$$
\theta[(s, \mathbf{x}),(t, \mathbf{y})]=\theta(4 \mathcal{I}(\mathbf{x}, \mathbf{y}), \mathbf{0})=(\hat{\theta}(4 \mathcal{I}(\mathbf{x}, \mathbf{y})), \mathbf{0})=(4 \hat{\theta}(\mathcal{I}(\mathbf{x}, \mathbf{y})), \mathbf{0})
$$

and

$$
[\theta(s, \mathbf{x}), \theta(t, \mathbf{y})]=[(*, \bar{\theta}(\mathbf{x})),(*, \bar{\theta}(\mathbf{y}))]=(4 \mathcal{I}(\bar{\theta}(\mathbf{x}), \bar{\theta}(\mathbf{y})), \mathbf{0})
$$

yield

$$
\mathcal{I}(\bar{\theta}(\mathbf{x}), \bar{\theta}(\mathbf{y}))=\hat{\theta}(\mathcal{I}(\mathbf{x}, \mathbf{y}))
$$


or, equivalently,

$$
\left(\bar{\theta}(\mathbf{x})^{t} J_{1} \bar{\theta}(\mathbf{y}), \bar{\theta}(\mathbf{x})^{t} J_{2} \bar{\theta}(\mathbf{y}), \bar{\theta}(\mathbf{x})^{t} J_{3} \bar{\theta}(\mathbf{y})\right)^{t}=\hat{\theta} \cdot\left(\mathbf{x}^{t} J_{1} \mathbf{y}, \mathbf{x}^{t} J_{2} \mathbf{y}, \mathbf{x}^{t} J_{3} \mathbf{y}\right)^{t}
$$

for all $\mathbf{x}, \mathbf{y}$. This happens if and only if $\bar{\theta}^{t} \mathbf{J} \bar{\theta}=\hat{\theta} \mathbf{J}$.

Conversely, suppose that $\bar{\theta} \in O(\mathbf{J}, 4)$, i.e., $\bar{\theta}^{t} \mathbf{J} \bar{\theta}=\lambda \mathbf{J}$ is satisfied for some $\lambda \in$ $\mathrm{GL}(3, \mathbb{R})$. We define $\theta \in \operatorname{Aut}\left(\mathfrak{N}_{2}\right)$ by

$$
\theta(s, \mathbf{x})=(\lambda \cdot s, \bar{\theta}(\mathbf{x})) .
$$

Then

$$
\begin{aligned}
\theta((s, \mathbf{x}) \cdot(t, \mathbf{y})) & =\theta(s+t+2 \mathcal{I}(\mathbf{x}, \mathbf{y}), \mathbf{x}+\mathbf{y}) \\
& =(\lambda \cdot(s+t+2 \mathcal{I}(\mathbf{x}, \mathbf{y})), \bar{\theta}(\mathbf{x}+\mathbf{y})) \\
& =(\lambda \cdot s+\lambda \cdot t+\lambda \cdot 2 \mathcal{I}(\mathbf{x}, \mathbf{y}), \bar{\theta}(\mathbf{x}+\mathbf{y})) \\
\theta(s, \mathbf{x}) \cdot \theta(t, \mathbf{y}) & =(\lambda \cdot s, \bar{\theta}(\mathbf{x})) \cdot(\lambda \cdot t, \bar{\theta}(\mathbf{y})) \\
& =(\lambda \cdot s+\lambda \cdot t+2 \mathcal{I}(\bar{\theta}(\mathbf{x}), \bar{\theta}(\mathbf{y})), \bar{\theta}(\mathbf{x})+\bar{\theta}(\mathbf{y})) .
\end{aligned}
$$

Now the condition $\bar{\theta}^{t} \mathbf{J} \bar{\theta}=\lambda \mathbf{J}$ guarantees that

$$
\lambda \cdot \mathcal{I}(\mathbf{x}, \mathbf{y})=\mathcal{I}(\bar{\theta}(\mathbf{x}), \bar{\theta}(\mathbf{y})) .
$$

Thus $\theta$ is an automorphism of $\mathfrak{N}_{2}$. Moreover, this defines a split homomorphism $O(\mathbf{J}, 4) \rightarrow \operatorname{Aut}\left(\mathfrak{N}_{2}\right)$.

Theorem 3.6 (Structure of Aut $\left(\mathfrak{N}_{2}\right)$ ).

$$
\begin{aligned}
\operatorname{Aut}\left(\mathfrak{N}_{2}\right) & =\operatorname{Hom}\left(\mathbb{R}^{4}, \mathbb{R}^{3}\right) \rtimes O(\mathbf{J}, 4) \\
& =\operatorname{Hom}\left(\mathbb{R}^{4}, \mathbb{R}^{3}\right) \rtimes\left(\left(S_{\ell}^{3} \times_{\mathbb{Z}_{2}} S_{r}^{3}\right) \times \mathbb{R}^{+}\right),
\end{aligned}
$$

where an element $(\eta, A) \in \operatorname{Hom}\left(\mathbb{R}^{4}, \mathbb{R}^{3}\right) \rtimes O(\mathbf{J}, 4)$ acts by

$$
(\eta, A)(s, \mathbf{x})=(\hat{A} s+\eta(\mathbf{x}), A \mathbf{x}) .
$$

Proof. Let $\theta \in \operatorname{Aut}\left(\mathfrak{N}_{2}\right)$. Then we have the following commutative diagram of exact sequences:

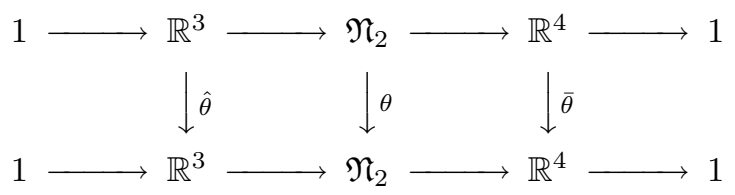

Thus $\theta(s, \mathbf{x})=(\hat{\theta}(s)+\eta(s, \mathbf{x}), \bar{\theta}(\mathbf{x}))$ for $(s, \mathbf{x}) \in \mathfrak{N}_{2}$, where $\eta: \mathfrak{N}_{2} \rightarrow \mathbb{R}^{3}$. Since $\theta$ is a homomorphism, one can show that $\eta$ is a homomorphism, i.e.,

$$
\eta((s, \mathbf{x})(t, \mathbf{y}))=\eta(s, \mathbf{x})+\eta(t, \mathbf{y}) .
$$

In particular, $(\hat{\theta}(s), \mathbf{0})=\theta(s, \mathbf{0})=(\hat{\theta}(s)+\eta(s, \mathbf{0}), \mathbf{0})$ implies that $\eta(s, \mathbf{0})=0$ for all $s \in \mathbb{R}^{3}$, and thus $\eta(s, \mathbf{x})=\eta((s, \mathbf{0})(0, \mathbf{x}))=\eta(s, \mathbf{0})+\eta(0, \mathbf{x})=\eta(0, \mathbf{x})$. Hence $\eta \in \operatorname{Hom}\left(\mathbb{R}^{4}, \mathbb{R}^{3}\right)$.

Let us find out the kernel of the surjective homomorphism of Lemma 3.5

$$
\operatorname{Aut}\left(\mathfrak{N}_{2}\right) \rightarrow O(\mathbf{J}, 4), \quad \theta \mapsto \bar{\theta} .
$$


Suppose that $\theta \in \operatorname{Aut}\left(\mathfrak{N}_{2}\right)$ with $\bar{\theta}=\operatorname{id}_{\mathbb{R}^{4}}$. Then $\hat{\theta}=\operatorname{id}_{\mathbb{R}^{3}}$ and thus $\theta(s, \mathbf{x})=$ $(s+\eta(\mathbf{x}), \mathbf{x})$ for some $\eta \in \operatorname{Hom}\left(\mathbb{R}^{4}, \mathbb{R}^{3}\right)$. Conversely given $\eta \in \operatorname{Hom}\left(\mathbb{R}^{4}, \mathbb{R}^{3}\right)$, define $\theta \in \operatorname{Aut}\left(\mathfrak{N}_{2}\right)$ by $\theta(s, \mathbf{x})=(s+\eta(\mathbf{x}), \mathbf{x})$. Clearly this $\theta$ lies in the kernel of the homomorphism. Hence we have a short exact sequence

$$
1 \rightarrow \operatorname{Hom}\left(\mathbb{R}^{4}, \mathbb{R}^{3}\right) \rightarrow \operatorname{Aut}\left(\mathfrak{N}_{2}\right) \rightarrow O(\mathbf{J}, 4) \rightarrow 1 .
$$

By Lemma 3.5 this sequence is split.

Note that $\operatorname{Hom}\left(\mathbb{R}^{4}, \mathbb{R}^{3}\right) \rtimes O(\mathbf{J}, 4)$ is sitting in $\operatorname{Hom}\left(\mathbb{R}^{4}, \mathbb{R}^{3}\right) \rtimes(\mathrm{GL}(3, \mathbb{R}) \times O(\mathbf{J}, 4))$ as $(\eta,(\hat{A}, A))$, and the action of $O(\mathbf{J}, 4)$ on $\operatorname{Hom}\left(\mathbb{R}^{4}, \mathbb{R}^{3}\right)$ is ${ }^{A} \eta(\mathbf{x})=\hat{A} \cdot \eta\left(A^{-1} \mathbf{x}\right)$. The group operation on $\mathfrak{N}_{2} \rtimes O(\mathbf{J}, 4)$ is given by

$$
\begin{aligned}
((s, \mathbf{x}), A)((t, \mathbf{y}), B) & =\left((s, \mathbf{x}) \cdot{ }^{A}(t, \mathbf{y}), A B\right) \\
& =((s, \mathbf{x}) \cdot(\hat{A} t, A \mathbf{y}), A B) \\
& =((s+\hat{A} t+2 \mathcal{I}(\mathbf{x}, A \mathbf{y}), \mathbf{x}+A \mathbf{y}), A B) .
\end{aligned}
$$

\section{The Structure of AB-Groups for $\mathbb{R}^{3} \tilde{\times} \mathbb{R}^{4}$}

Let $\Pi \subset \mathfrak{N}_{2} \rtimes \operatorname{Aut}\left(\mathfrak{N}_{2}\right)$ be an AB-group. Then it is well known that $\Gamma=\Pi \cap \mathfrak{N}_{2}$, the pure translations in $\Pi$, is the maximal normal nilpotent subgroup, and $\Phi=$ $\Pi / \Gamma$, the holonomy group of $\Pi$, is finite. Since $\Gamma$ is a lattice of $\mathfrak{N}_{2}, \Gamma \cap \mathcal{Z}\left(\mathfrak{N}_{2}\right)$ is a lattice of $\mathcal{Z}\left(\mathfrak{N}_{2}\right)=\mathbb{R}^{3}$, and $\Gamma / \Gamma \cap \mathcal{Z}\left(\mathfrak{N}_{2}\right)$ is a lattice of $\mathfrak{N}_{2} / \mathcal{Z}\left(\mathfrak{N}_{2}\right)=\mathbb{R}^{4}$. Thus $\Gamma \cap \mathcal{Z}\left(\mathfrak{N}_{2}\right) \cong \mathbb{Z}^{3}$ and $\Gamma / \Gamma \cap \mathcal{Z}\left(\mathfrak{N}_{2}\right) \cong \mathbb{Z}^{4}$.

Consider the following natural commutative diagram:

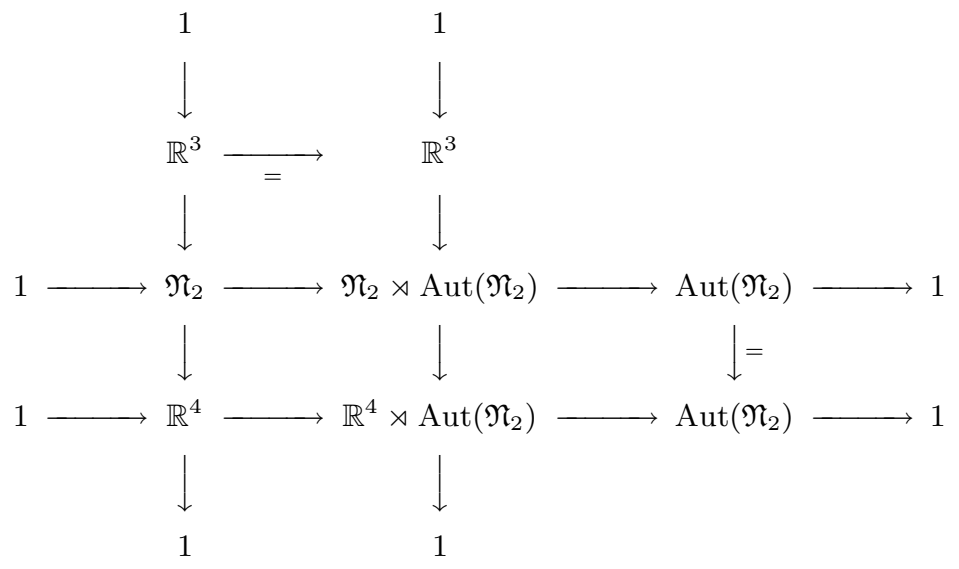

Recall from Theorem 3.6 that an element $(\eta, A) \in \operatorname{Aut}\left(\mathfrak{N}_{2}\right)=\operatorname{Hom}\left(\mathbb{R}^{4}, \mathbb{R}^{3}\right) \rtimes$ $O(\mathbf{J}, 4)$ acts on $(s, \mathbf{x}) \in \mathfrak{N}_{2}$ by

$$
(\eta, A)(s, \mathbf{x})=(\hat{A} s+\eta(\mathbf{x}), A \mathbf{x}) .
$$

Thus $O(\mathbf{J}, 4)$ acts on $\mathbb{R}^{3}$ via the homomorphism ${ }^{\wedge}: O(\mathbf{J}, 4) \rightarrow \mathrm{GL}(3, \mathbb{R})$, and $O(\mathbf{J}, 4)$ acts on $\mathbb{R}^{4}$ by matrix multiplication $O(\mathbf{J}, 4) \times \mathbb{R}^{4} \rightarrow \mathbb{R}^{4}$. 
Let $Q=\Pi / \mathbb{Z}^{3}$. Then the previous diagram induces the following commutative diagram:

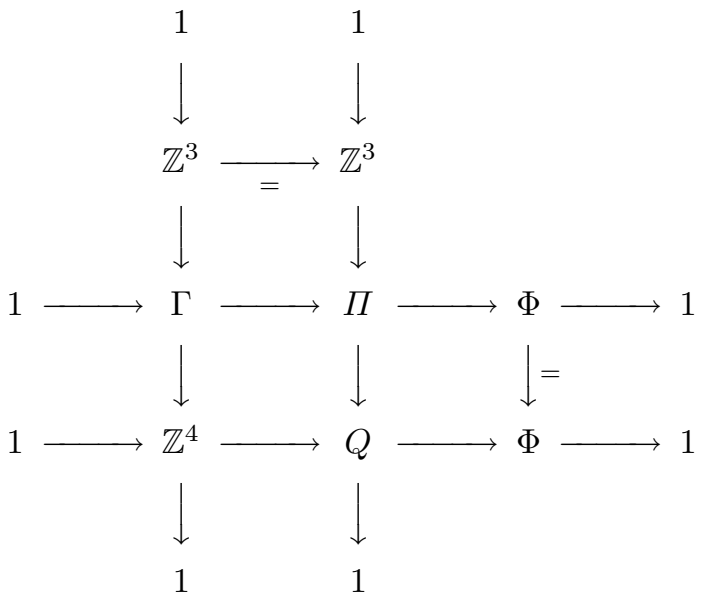

Here $\Phi \subset O(\mathbf{J}, 4)$ acts on $\mathbb{Z}^{4}$ by matrix multiplication, and on $\mathbb{Z}^{3}$ via the homomorphism ^ $: O(\mathbf{J}, 4) \rightarrow \mathrm{GL}(3, \mathbb{R})$.

Lemma 4.1. Let $\mathbf{v}_{1}, \mathbf{v}_{2}, \mathbf{v}_{3}, \mathbf{v}_{4}$ be a basis of $\mathbb{R}^{4}$ with rational entries. Then for each $i=1,2,3,4$, the set $\left\{\mathcal{I}\left(\mathbf{v}_{i}, \mathbf{v}_{j}\right) \mid j=1, \cdots, \widehat{i}, \cdots, 4\right\}$ forms a lattice of $\mathbb{R}^{3}$.

Proof. Let $V$ be the $4 \times 4$-matrix whose column vectors are $\mathbf{v}_{1}, \mathbf{v}_{2}, \mathbf{v}_{3}, \mathbf{v}_{4}$, and let $W_{i}$ be the $3 \times 3$-matrix whose column vectors are $\mathcal{I}\left(\mathbf{v}_{i}, \mathbf{v}_{j}\right)$ with $j=1, \cdots, \hat{i}, \cdots, 4$. Then we can show that

$$
\operatorname{det}\left(W_{1}\right)=\operatorname{det}\left[\mathcal{I}\left(\mathbf{v}_{1}, \mathbf{v}_{2}\right), \mathcal{I}\left(\mathbf{v}_{1}, \mathbf{v}_{3}\right), \mathcal{I}\left(\mathbf{v}_{1}, \mathbf{v}_{4}\right)\right]=-\left|\mathbf{v}_{1}\right|^{2} \operatorname{det}\left[\mathbf{v}_{1} \mathbf{v}_{2} \mathbf{v}_{3} \mathbf{v}_{4}\right] .
$$

Similarly, $\operatorname{det}\left(W_{i}\right)= \pm\left|\mathbf{v}_{i}\right|^{2} \operatorname{det}\left[\mathbf{v}_{1} \mathbf{v}_{2} \mathbf{v}_{3} \mathbf{v}_{4}\right]$. Thus if $\mathbf{v}_{1}, \mathbf{v}_{2}, \mathbf{v}_{3}, \mathbf{v}_{4}$ is a basis of $\mathbb{R}^{4}$, then the set $\left\{\mathcal{I}\left(\mathbf{v}_{i}, \mathbf{v}_{j}\right) \mid j=1, \cdots, \hat{i}, \cdots, 4\right\}$ spans $\mathbb{R}^{3}$. The map $\mathcal{I}(\mathbf{x}, \mathbf{y})$ consists of polynomial functions of the entries of $\mathbf{x}$ and $\mathbf{y}$. Therefore the group generated by $\left\{\mathcal{I}\left(\mathbf{v}_{i}, \mathbf{v}_{j}\right) \mid j=1, \cdots, \widehat{i}, \cdots, 4\right\}$ is discrete so that it forms a lattice of $\mathbb{R}^{3}$.

This lemma tells us that the lattice $\mathbb{Z}^{4}$ of $\mathbb{R}^{4}$ generated by $\left\{\mathbf{v}_{1}, \mathbf{v}_{2}, \mathbf{v}_{3}, \mathbf{v}_{4}\right\}$ yields a lattice generated by $\left\{4 \mathcal{I}\left(\mathbf{v}_{i}, \mathbf{v}_{j}\right) \mid j=1, \cdots, \widehat{i}, \cdots, 4\right\}$, which must be contained in $\mathbb{Z}^{3}$ in the above diagram. However, the $\mathbb{Z}^{3}$ in the diagram can be finer than the lattice generated by $\left\{4 \mathcal{I}\left(\mathbf{v}_{i}, \mathbf{v}_{j}\right) \mid j=1, \cdots, \widehat{i}, \cdots, 4\right\}$.

Recall from [6, Proposition 2] that a virtually free abelian group $1 \rightarrow \mathbb{Z}^{4} \rightarrow Q \rightarrow$ $\Phi \rightarrow 1$ is a crystallographic group if and only if the centralizer of $\mathbb{Z}^{4}$ in $Q$ has no torsion elements. Since $\Phi$ acts effectively on $\mathbb{Z}^{4}$, it follows that $Q$ is naturally a 4-dimensional crystallographic group.

The finite group $\Phi$ must be in a maximal compact subgroup $\mathrm{SO}(4)$ of $\operatorname{Aut}\left(\mathfrak{N}_{2}\right)=$ $\operatorname{Hom}\left(\mathbb{R}^{4}, \mathbb{R}^{3}\right) \rtimes\left(\mathrm{SO}(4) \times \mathbb{R}^{+}\right)$. Note that this coincides with the compact subgroup $\operatorname{Sp}(1) \times_{\mathbb{Z}_{2}} \operatorname{Sp}(1)$ in $\operatorname{Isom}_{0}\left(\mathfrak{N}_{2}\right)=\mathfrak{N}_{2} \rtimes\left(\operatorname{Sp}(1) \times_{\mathbb{Z}_{2}} \operatorname{Sp}(1)\right)=S^{3} \times_{\mathbb{Z}_{2}} S^{3}=\mathfrak{N}_{2} \rtimes \mathrm{SO}(4)$. See Theorem 2.1.

Construction of an AB-group $\Pi$ from $Q$. For each 4-dimensional crystallographic group $Q$, we shall check if there exists an $\mathrm{AB}$-group $\Pi$ constructed from $Q$; that is, a torsion free $\Pi \subset \mathfrak{N}_{2} \rtimes \mathrm{SO}(4) \subset \mathfrak{N}_{2} \rtimes \operatorname{Aut}\left(\mathfrak{N}_{2}\right)$ fitting the short exact sequence

$$
1 \longrightarrow \mathbb{Z}^{3} \longrightarrow \Pi \longrightarrow Q \longrightarrow 1 \text {. }
$$


This is the key notion for our arguments and construction. We have a complete classification of 4-dimensional crystallographic groups ( $Q$ 's in the above statement). We shall use the representations of the 4-dimensional crystallographic groups given in the book [1]. Every $Q$ has an explicit representation $Q \longrightarrow \mathbb{R}^{4} \rtimes \mathrm{GL}(4, \mathbb{Z}$ ) (not into $\left.\mathbb{R}^{4} \rtimes O(4)\right)$ in this book.

\section{Algebraic CRiteria fOR eXistence of lifting}

Corollary 5.1. For a 4-dimensional crystallographic group $Q$ to have an AB-group $\Pi$, its holonomy group $\Phi$ must be in $\mathrm{SO}(4)$. Therefore, if $\Phi$ contains a matrix of determinant -1 , there is no AB-group $\Pi$ from $Q$.

Proof. The holonomy group $\Phi$ is a finite subgroup of $O(\mathbf{J}, 4)=\mathrm{SO}(4) \times \mathbb{R}^{+}$(by Proposition 3.2 so that $\Phi \subset \mathrm{SO}(4)$.

Our goal is to determine which $Q$ will give rise to a torsion free $\Pi$ that fits the diagram (4-1). When $Q$ is torsion free, then $\Pi$ will be automatically torsion free, but when $Q$ contains a torsion subgroup $Q_{0}$, we need to check whether the lift $Q_{0}$ to $\Pi$ will be torsion free.

Let $\Pi_{0}$ be the lift of $Q_{0}$ to $\Pi$. Thus $1 \rightarrow \mathbb{Z}^{3} \rightarrow \Pi_{0} \rightarrow Q_{0} \rightarrow 1$ is exact. The finite subgroup $Q_{0} \subset Q$ injects into the holonomy group (of $Q$ ) $\Phi \subset \mathrm{SO}(4)$ so that we can view $Q_{0} \subset \mathrm{SO}(4)$. Recall $\mathrm{SO}(4)=S_{\ell}^{3} \times_{\mathbb{Z}_{2}} S_{r}^{3}$, and $S_{\ell}^{3}$ is the kernel of the homomorphism

$$
\epsilon: \mathrm{SO}(4) \longrightarrow \mathrm{SO}(3) \text {. }
$$

Let $Q_{1}=Q_{0} \cap S_{\ell}^{3}$, and $Q_{2}=Q_{0} / Q_{1}$ so that

$$
1 \rightarrow Q_{1} \rightarrow Q_{0} \rightarrow Q_{2} \rightarrow 1
$$

is exact. Consider the pullbacks of the extension $1 \rightarrow \mathbb{Z}^{3} \rightarrow \Pi \rightarrow Q \rightarrow 1$ by the inclusions $Q_{1} \hookrightarrow Q_{0} \hookrightarrow Q$. Then we have the following commutative diagram:

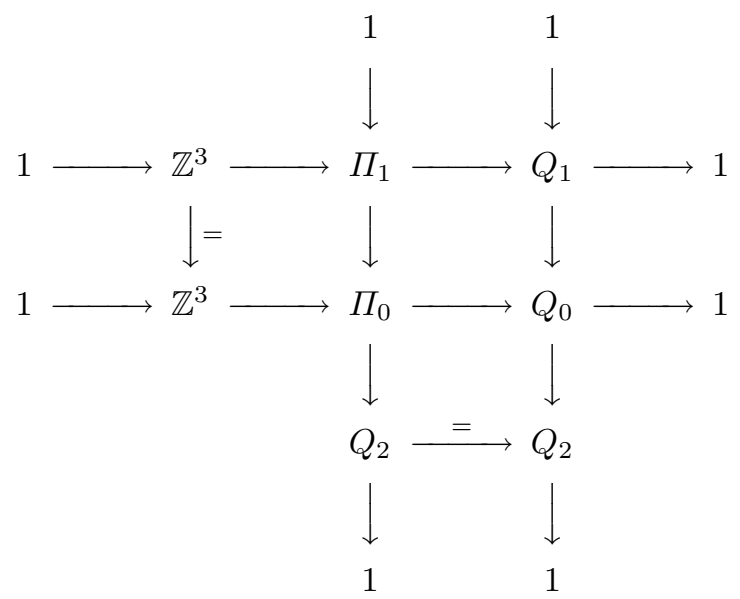

Since $Q_{1}$ acts trivially on $\mathbb{Z}^{3}, \Pi_{1}=C_{\Pi_{0}}\left(\mathbb{Z}^{3}\right)$, the centralizer of $\mathbb{Z}^{3}$ in $\Pi_{0}$, is torsion free. Hence by [6. Proposition 2], $\Pi_{1}=C_{\Pi_{0}}\left(\mathbb{Z}^{3}\right) \cong \mathbb{Z}^{3}$ and $Q_{1}=\Pi_{1} / \mathbb{Z}^{3} \subset S^{3}$ is abelian. But every abelian subgroup of $S^{3}$ is cyclic. Hence $Q_{1}$ is cyclic. Therefore, $Q_{0}$ is a central extension of $Q_{1}$ by $Q_{2}$.

Since $Q_{2}$ acts effectively on $\mathbb{Z}^{3}, \Pi_{0}$ is naturally a 3 -dimensional orientable Bieberbach group, and thus $Q_{2}$ must be a holonomy group, i.e, $Q_{2} \cong 1, \mathbb{Z}_{2}, \mathbb{Z}_{3}, \mathbb{Z}_{4}, \mathbb{Z}_{6}$ or $\mathbb{Z}_{2} \times \mathbb{Z}_{2}$. 
In particular, we have shown the following.

Lemma 5.2. Any finite subgroup of $Q$ is an extension of a cyclic group by an orientable 3 -dimensional holonomy group $1, \mathbb{Z}_{2}, \mathbb{Z}_{3}, \mathbb{Z}_{4}, \mathbb{Z}_{6}$ or $\mathbb{Z}_{2} \times \mathbb{Z}_{2}$, where the cyclic group can be conjugated into $S_{\ell}^{3}$.

Lemma 5.3. $Q$ cannot contain a subgroup isomorphic to $\mathbb{Z}_{2} \times \mathbb{Z}_{2} \times \mathbb{Z}_{2}$.

Proof. Suppose $Q \subset \mathbb{R}^{4} \rtimes \mathrm{SO}(4)$ contains a finite subgroup $Q_{0}$ isomorphic to $\mathbb{Z}_{2} \times$ $\mathbb{Z}_{2} \times \mathbb{Z}_{2}$. Then $Q_{0}$ inject into $\Phi$. By Lemma 5.2, $Q_{0}$ is an extension of $Q_{1}$ by $Q_{2}$, where $Q_{0}, Q_{1}, Q_{2}$ fit into the commutative diagram (5-1), $Q_{1} \cong \mathbb{Z}_{2}$ acts trivially on $\mathbb{Z}^{3}$ and $Q_{2} \cong \mathbb{Z}_{2} \times \mathbb{Z}_{2}$ acts effectively on $\mathbb{Z}^{3}$. By conjugation we may assume that $\Pi_{0}$ is the group $\mathfrak{G}_{6}$ of [9, Theorem 3.5.5]. Thus $Q_{0}$ is a finite group acting freely on the 3 -torus $T$ so that $T / Q_{0}$ is homeomorphic to $\mathbb{R}^{3} / \mathfrak{G}_{6}$. Since $Q_{0}$ is an abelian group, by [7, Theorem 6.1] or [3, Corollary 5.3], $Q_{0}$ must be $\mathbb{Z}_{2} \times \mathbb{Z}_{2}, \mathbb{Z}_{4} \times \mathbb{Z}_{2}$ or $\mathbb{Z}_{4} \times \mathbb{Z}_{4}$. This contradicts the assumption that $Q_{0} \cong \mathbb{Z}_{2} \times \mathbb{Z}_{2} \times \mathbb{Z}_{2}$.

The following is crucial in eliminating the possibility of constructing a Bieberbach group $\Pi$ from a crystallographic group $Q$.

Proposition 5.4. Let $Q$ be an abstract orientable crystallographic group (not yet embedded into $\mathbb{R}^{4} \rtimes \mathrm{SO}(4)$ ) with holonomy group $\Phi$. Then $\Phi$ contains unique subgroups $\Phi_{\ell}$ and $\Phi_{r}$ (up to interchanging) with the property that

(1) $\Phi_{\ell} \cap \Phi_{r} \subset\{ \pm I\}$.

(2) Elements from $\Phi_{\ell}$ and $\Phi_{r}$ commute with each other.

(3) Suppose $Q_{0}$ in the diagram (5-1) is obtained from $Q$. Then either $Q_{1}=$ $Q_{0} \cap \Phi_{\ell}$ or $Q_{1}=Q_{0} \cap \Phi_{r}$.

Proof. Recall from Lemma 3.4 that $S_{\ell}^{3} \cup S_{r}^{3}$ is exactly the subset of $\mathrm{SO}(4)$ consisting of elements with eigenvalues $a+b \mathbf{i}, a+b \mathbf{i}, a-b \mathbf{i}, a-b \mathbf{i}$ with $b \neq 0$, together with $\pm I$. In fact, $\pm I \in S_{\ell}^{3} \cap S_{r}^{3}$. Assume $\Phi$ is embedded in $\mathrm{SO}(4)$ in any way. Then let

$$
\begin{aligned}
& \Phi_{\ell}=\Phi \cap S_{\ell}^{3}, \\
& \Phi_{r}=\Phi \cap S_{r}^{3} .
\end{aligned}
$$

The choice of an embedding does not change the sets $\Phi_{\ell}$ and $\Phi_{r}$ except interchanging, because conjugation leaves the eigenvalues unchanged (this happens if $\Phi \subset \mathrm{SO}(4)$ is conjugated by the matrix $\sigma$ in $(2-2))$. The last claim is clear because the kernel of $\epsilon: O(\mathbf{J}, 4) \longrightarrow \mathrm{SO}(3) \times \mathbb{R}^{+}$is $S_{\ell}^{3}$.

Procedure 5.5 (of finding $\Phi_{\ell}$ and $\Phi_{r}$ from $Q$ ). We assume $\Phi$ lies in $\operatorname{GL}(4, \mathbb{R}$ ). Look at those elements of $\Phi$ which are not $\pm I$ (since $\pm I$ must belong to both $\Phi_{\ell}$ and $\Phi_{r}$ ). Suppose $A \in \Phi$ has the eigenvalues $a \pm b \mathbf{i}$, with $b \neq 0$, of multiplicity 2 . Then put $A$ into $\Phi_{\ell}$. For the next $B \in \Phi$ with the right eigenvalues, we look at the product $A B$. If this has the right eigenvalues, then put $B$ into $\Phi_{\ell}$. Otherwise put $B$ into $\Phi_{r}$. Proceed to the next element.

Corollary 5.6. Suppose $Q$ contains a non-abelian torsion subgroup $Q_{0}$ of order $>12$, with all the matrices of $Q_{0}$ lying completely inside $\Phi_{\ell}$ or $\Phi_{r}$. Then $Q$ does not yield a Bieberbach group $\Pi$.

Proof. If $Q_{0} \subset \Phi_{\ell}$, then $Q_{0}=Q_{1}$. But this is impossible since $Q_{0}$ is not abelian. Thus we must have $Q_{0} \subset \Phi_{r}$. Then $Q_{1}$ is at most $\mathbb{Z}_{2}$ (or trivial) so that $Q_{2}$ has order $>6$. But the order of $Q_{2}$ must be $\leq 6$ by Lemma [5.2] a contradiction. 
Corollary 5.7. Suppose $Q$ contains a non-abelian torsion subgroup $Q_{0}$ of order $p$, and let the highest order of holonomy matrices in $\Phi_{\ell} \cup \Phi_{r}$ be $r$. If $p / r>6$, then $Q$ does not yield a Bieberbach group $\Pi$.

Proof. Since the kernel $Q_{1}$ of the map $\epsilon$ must be cyclic coming from $\Phi_{\ell}$ or $\Phi_{r}, Q_{2}$ will have order $>6$. Now apply Lemma 5.2

In what follows, the bold-faced numbers associated to the 4-dimensional crystallographic groups refer to the numbering in the book [1].

Corollary 5.8. There is no AB-group $\Pi \subset \mathfrak{N}_{2} \rtimes \operatorname{Aut}\left(\mathfrak{N}_{2}\right)$ constructed from $Q$ given in 32/12/02/004 (holonomy group of order 64).

Proof. Let $Q_{0}$ be the subgroups of $Q$

$$
Q_{0}=\left\langle t_{2}(a, A)(b, B)(d, D),(a, A)^{7}(b, B)(c, C)(d, D)\right\rangle,
$$

a non-abelian group of order 16. By looking at the eigenvalues, we find that $\Delta=$ $Q_{0} \cap\left(\Phi_{\ell} \cup \Phi_{r}\right)$ is a non-cyclic group of order 8. Further, $\Delta$ is completely in one side of $\Phi_{\ell} \cup \Phi_{r}$ (again from the eigenvalues). Since $\Delta$ is not cyclic, it cannot be in the kernel of $\epsilon$. Therefore, $Q_{1}=\left\{ \pm I_{4}\right\}$ and $Q_{2}$ is a group of order 8, which is impossible by Lemma 5.2 .

Recall that any almost Bieberbach group $\Pi$ for $\mathfrak{N}_{2}$ is a torsion free extension of $\mathbb{Z}_{3}$ by a 4-dimensional crystallographic group $Q$. There are 4783 4-dimensional crystallographic groups up to isomorphism. However, not all 4-dimensional crystallographic groups are qualified here. By Corollary 5.1, we eliminate about half of those 4-dimensional crystallographic groups. By Lemmas 5.2 5.3, and Corollaries 5.6 and 5.7 together with Lemma 3.4 we can eliminate most of the remaining 4 -dimensional crystallographic groups with holonomy order $\leq 48$, except for a few cases.

\section{Elimination of SPECial CASES}

In this section, we shall prove that there is no construction of AB-groups from the crystallographic groups of type $\mathbf{3 3 / 0 8}$ (and 33/12), 33/09 and 33/10. This requires special effort.

Given a 4-dimensional crystallographic group $Q$, it may happen that there is no AB-group $\Pi$ constructed from $Q$ itself, but there may be one from a conjugate of $Q$. But, it turns out that we need to check only two cases of different representations of $Q$ into $E_{0}(4)=\mathbb{R}^{4} \rtimes \mathrm{SO}(4)$ in this special case.

First, we present an example of $Q$, which has two distinct (non-isomorphic) constructions depending on the representations:

Example 6.1. Two affinely conjugate representations of the same group into $E_{0}(4)$ as crystallographic groups can result in two non-isomorphic constructions of $\Pi$.

Consider the 4-dimensional crystallographic group $Q$ given by

$$
Q=\left\langle\left(\mathbf{e}_{1}, I\right),\left(\mathbf{e}_{2}, I\right),\left(\mathbf{e}_{3}, I\right),\left(\mathbf{e}_{4}, I\right),\left(\mathbf{0},-K_{1}\right)\right\rangle .
$$

This is the group given in $\mathbf{1 0 / 0 1}$. Conjugation by $\sigma$ (see $(2-2)$ ) sends $\left(\mathbf{e}_{1}, I\right)$ to $\left(-\mathbf{e}_{1}, I\right),\left(\mathbf{e}_{i}, I\right)$ to $\left(\mathbf{e}_{i}, I\right)$ for $i=2,3,4$. Also it sends $-K_{1}$ to $-J_{1}$. Thus, the conjugation maps $Q$ to

$$
Q^{\prime}=\left\langle\left(\mathbf{e}_{1}, I\right),\left(\mathbf{e}_{2}, I\right),\left(\mathbf{e}_{3}, I\right),\left(\mathbf{e}_{4}, I\right),\left(\mathbf{0},-J_{1}\right)\right\rangle .
$$


Now consider the two groups

$$
\begin{aligned}
\Pi & =\left\langle\left(0, \mathbf{e}_{1}, I\right),\left(0, \mathbf{e}_{2}, I\right),\left(0, \mathbf{e}_{3}, I\right),\left(0, \mathbf{e}_{4}, I\right),\left(a, \mathbf{0},-K_{1}\right)\right\rangle, \\
\Pi^{\prime} & =\left\langle\left(0, \mathbf{e}_{1}, I\right),\left(0, \mathbf{e}_{2}, I\right),\left(0, \mathbf{e}_{3}, I\right),\left(0, \mathbf{e}_{4}, I\right),\left(a, \mathbf{0},-J_{1}\right)\right\rangle,
\end{aligned}
$$

where $a=[1,0,0]^{t}$. It is quite easy to see that the subgroup $\Gamma$ generated by the 4 translations is a lattice of the Heisenberg group (of rank 7), and is the Fitting subgroup (the maximal normal nilpotent subgroup) in both $\Pi$ and $\Pi^{\prime}$. Further,

$$
\Gamma \cap \mathbb{R}^{3} \cong \mathbb{Z}^{3}
$$

is generated by the 3 vectors $[4,0,0]^{t},[0,4,0]^{t},[0,0,4]^{t}$. Now the action of the holonomy group on this characteristic subgroup $\mathbb{Z}^{3}$ is via

$$
\widehat{J}_{1}=\left[\begin{array}{rrr}
1 & 0 & 0 \\
0 & -1 & 0 \\
0 & 0 & -1
\end{array}\right], \quad \widehat{K}_{1}=\left[\begin{array}{lll}
1 & 0 & 0 \\
0 & 1 & 0 \\
0 & 0 & 1
\end{array}\right] .
$$

Therefore, it is easy to see now that both $\Pi$ and $\Pi^{\prime}$ are torsion free. In fact, in both cases,

$$
\left(a, \mathbf{0},-J_{1}\right)^{4}=\left([4,0,0]^{t}, \mathbf{0}, I\right)=\left(a, \mathbf{0},-K_{1}\right)^{4} .
$$

Thus, they are AB-groups. However, they cannot be isomorphic because the action of the holonomy groups on the center of the Fitting subgroups $\Gamma$ are distinct as shown above. Recall that $Q^{\prime}$ was conjugate to $Q$ by an element of $O(4)$.

Proposition 6.2. Let $Q \subset \mathbb{R}^{4} \rtimes \mathrm{SO}(4)$ be a crystallographic group with holonomy group $\Phi$. Suppose that any symmetric matrix that commutes with every element of $\Phi$ has the form $\lambda I$, where $\lambda \neq 0$. Then there is an $A B$-group $\Pi \subset \mathfrak{N}_{2} \rtimes \operatorname{Aut}\left(\mathfrak{N}_{2}\right)$ constructed from some embedding $Q$ into $\mathbb{R}^{4} \rtimes \mathrm{SO}(4)$ if and only if there is an $A B$-group $\Pi$ constructed from $Q$ or $(\mathbf{0}, \sigma)^{-1} Q(\mathbf{0}, \sigma)$.

Proof. Suppose that there is an AB-group $\Pi$ constructed from some $Q^{\prime}$, an embedding of $Q$ into $\mathbb{R}^{4} \rtimes \mathrm{SO}(4)$. Then, by the second Bieberbach Theorem, $Q^{\prime}$ is a conjugate to $Q$ by an element of $\mathbb{R}^{4} \rtimes \mathrm{GL}(4, \mathbb{R})$, say, $(\mathbf{x}, X)$. Then

$$
\begin{aligned}
& (\mathrm{x}, X)^{-1} Q(\mathrm{x}, X)=Q^{\prime} \subset \mathbb{R}^{4} \rtimes \mathrm{SO}(4) \\
\Longrightarrow & X^{-1} A X \in \mathrm{SO}(4) \text { for all } A \in \Phi \\
\Longleftrightarrow & \left(X^{-1} A X\right)\left(X^{-1} A X\right)^{t}=I_{4} \text { for all } A \in \Phi \\
\Longleftrightarrow & A S A^{t}=S \text { for all } A \in \Phi,
\end{aligned}
$$

where $S=X X^{t}$. By the assumption, $S=k I_{4}$ with $k>0$. Hence $X \in O(4) \times \mathbb{R}^{+}$.

If $X \in \mathrm{SO}(4) \times \mathbb{R}^{+}$, then conjugation of $\Pi$ by $(0, \mathbf{x}, X)^{-1}$ is an AB-group constructed from $Q$ because $(\mathbf{x}, X) Q^{\prime}(\mathbf{x}, X)^{-1}=Q$.

If $X \notin \mathrm{SO}(4) \times \mathbb{R}^{+}$, then $X=\sigma Y$ for some $Y \in \mathrm{SO}(4) \times \mathbb{R}^{+}$. Then

$$
(0, \sigma)(\sigma \mathbf{x}, Y) Q^{\prime}(\sigma \mathbf{x}, Y)^{-1}(0, \sigma)^{-1}=(\mathbf{x}, X) Q^{\prime}(\mathbf{x}, X)^{-1}=Q
$$

so that

$$
(\sigma \mathbf{x}, Y) Q^{\prime}(\sigma \mathbf{x}, Y)^{-1}=(0, \sigma)^{-1} Q(0, \sigma) .
$$

Therefore $(\sigma \mathbf{x}, Y) Q^{\prime}(\sigma \mathbf{x}, Y)^{-1}$ is an AB-group $\Pi$ constructed from $(\mathbf{0}, \sigma)^{-1} Q(\mathbf{0}, \sigma)$. The converse is trivial. 
Proposition 6.3. Let $Q \subset \mathbb{R}^{4} \rtimes \mathrm{SO}(4)$ be a crystallographic group generated by

$$
\left(\mathbf{v}_{1}, I\right),\left(\mathbf{v}_{2}, I\right),\left(\mathbf{v}_{3}, I\right),\left(\mathbf{v}_{4}, I\right),\left(\mathbf{a}_{1}, A_{1}\right),\left(\mathbf{a}_{2}, A_{2}\right), \cdots,\left(\mathbf{a}_{p}, A_{p}\right),
$$

where the subgroup $\left\langle\left(\mathbf{v}_{1}, I\right),\left(\mathbf{v}_{2}, I\right),\left(\mathbf{v}_{3}, I\right),\left(\mathbf{v}_{4}, I\right)\right\rangle$ is the maximal normal free abelian of rank 4 . Then there exists an AB-group obtained from $Q$ if and only if the group $\Pi_{\left(s_{1}, s_{2}, \cdots, s_{p}\right)}$ generated by

$$
\begin{gathered}
\left(0, \mathbf{v}_{1}, I\right),\left(0, \mathbf{v}_{2}, I\right),\left(0, \mathbf{v}_{3}, I\right),\left(0, \mathbf{v}_{4}, I\right), \\
\left(s_{1}, \mathbf{a}_{1}, A_{1}\right),\left(s_{2}, \mathbf{a}_{2}, A_{2}\right), \cdots,\left(s_{p}, \mathbf{a}_{p}, A_{p}\right)
\end{gathered}
$$

(for some $s_{1}, s_{2}, \cdots, s_{p} \in \mathbb{R}^{3}$ ) is an AB-group.

Proof. Any AB-group $\Pi \subset \mathfrak{N}_{2} \rtimes \operatorname{Aut}\left(\mathfrak{N}_{2}\right)$ obtained from $Q$ is generated by

$$
\begin{gathered}
\left(v_{1}, \mathbf{0}, I\right),\left(v_{2}, \mathbf{0}, I\right),\left(v_{3}, \mathbf{0}, I\right), \\
\left(w_{1}, \mathbf{v}_{1}, I\right),\left(w_{2}, \mathbf{v}_{2}, I\right),\left(w_{3}, \mathbf{v}_{3}, I\right),\left(w_{4}, \mathbf{v}_{4}, I\right), \\
\left(t_{1}, \mathbf{a}_{1}, A_{1}\right),\left(t_{2}, \mathbf{a}_{2}, A_{2}\right), \cdots,\left(t_{p}, \mathbf{a}_{p}, A_{p}\right)
\end{gathered}
$$

for some $v_{1}, v_{2}, v_{3}, w_{1}, w_{2}, w_{3}, w_{4}, t_{1}, t_{2}, \cdots, t_{p} \in \mathbb{R}^{3}$. Since the group generated by

$$
\left(w_{1}, \mathbf{v}_{1}, I\right),\left(w_{2}, \mathbf{v}_{2}, I\right),\left(w_{3}, \mathbf{v}_{3}, I\right),\left(w_{4}, \mathbf{v}_{4}, I\right)
$$

becomes a lattice of $\mathfrak{N}_{2}$ already (see Lemma 4.11), the group generated by

$$
\begin{gathered}
\left(w_{1}, \mathbf{v}_{1}, I\right),\left(w_{2}, \mathbf{v}_{2}, I\right),\left(w_{3}, \mathbf{v}_{3}, I\right),\left(w_{4}, \mathbf{v}_{4}, I\right), \\
\left(t_{1}, \mathbf{a}_{1}, A_{1}\right),\left(t_{2}, \mathbf{a}_{2}, A_{2}\right), \cdots,\left(t_{p}, \mathbf{a}_{p}, A_{p}\right)
\end{gathered}
$$

(without $\left.\left(v_{1}, \mathbf{0}, I\right),\left(v_{2}, \mathbf{0}, I\right),\left(v_{3}, \mathbf{0}, I\right)\right)$ will be a subgroup of $\Pi$ of finite index, and is an AB-group. Let us call this smaller group $\Pi^{\prime}$.

Let $(s, \mathbf{x}, \eta, E) \in \mathbb{R}^{3} \tilde{\times} \mathbb{R}^{4} \rtimes\left(\operatorname{Hom}\left(\mathbb{R}^{4}, \mathbb{R}^{3}\right) \rtimes O(\mathbf{J}, 4)\right)=\mathfrak{N}_{2} \rtimes \operatorname{Aut}\left(\mathfrak{N}_{2}\right)$. Then for $\left(0, \mathbf{v}_{i}\right) \in \mathbb{R}^{3} \tilde{\times} \mathbb{R}^{4}=\mathfrak{N}_{2}$,

$$
(s, \mathbf{x}, \eta, E)\left(0, \mathbf{v}_{i}\right)(s, \mathbf{x}, \eta, E)^{-1}=\left(\eta\left(\mathbf{v}_{i}\right)+4 \mathcal{I}\left(\mathbf{x}, E \mathbf{v}_{i}\right), E \mathbf{v}_{i}\right) .
$$

Thus given $w_{1}, w_{2}, w_{3}, w_{4} \in \mathbb{R}^{3}$, it is obvious that there is $\eta \in \operatorname{Hom}\left(\mathbb{R}^{4}, \mathbb{R}^{3}\right)$ such that $\eta\left(\mathbf{v}_{i}\right)=w_{i}$ for $i=1,2,3,4$. Hence $\tilde{\eta}=(0, \mathbf{0}, \eta, I) \in \mathfrak{N}_{2} \rtimes \operatorname{Aut}\left(\mathfrak{N}_{2}\right)$ and $\mu(\tilde{\eta})\left(w_{i}, \mathbf{v}_{i}, I\right)=\left(0, \mathbf{v}_{i}, I\right)$ for all $i=1,2,3,4$. Consequently, the group $\Pi^{\prime \prime}$ obtained by conjugating $\Pi^{\prime}$ by $\tilde{\eta}$ is another AB-group, satisfying the condition $w_{1}=\cdots=$ $w_{4}=0$. [In the course of conjugation, $t_{i}$ will change.]

Consequently, we have obtained an AB-group $\Pi^{\prime \prime}=\Pi_{\left(s_{1}, s_{2}, \cdots, s_{p}\right)} \subset \mathfrak{N}_{2} \rtimes$ $\operatorname{Aut}\left(\mathfrak{N}_{2}\right)$ generated by

$$
\begin{gathered}
\left(0, \mathbf{v}_{1}, I\right),\left(0, \mathbf{v}_{2}, I\right),\left(0, \mathbf{v}_{3}, I\right),\left(0, \mathbf{v}_{4}, I\right), \\
\left(s_{1}, \mathbf{a}_{1}, A_{1}\right),\left(s_{2}, \mathbf{a}_{2}, A_{2}\right), \cdots,\left(s_{p}, \mathbf{a}_{p}, A_{p}\right)
\end{gathered}
$$

for some $s_{1}, s_{2}, \cdots, s_{p} \in \mathbb{R}^{3}$.

The proposition says that if there is an $\mathrm{AB}$-group $\Pi$ constructed from $Q$, then $\Pi_{\left(s_{1}, s_{2}, \cdots, s_{p}\right)}$ is a subgroup of $\Pi$ of finite index, which is another AB-group constructed from $Q$. Conversely, if $\Pi_{\left(s_{1}, s_{2}, \cdots, s_{p}\right)}$ is an AB-group (i.e., is torsion free), then we are done. Therefore, the existence/non-existence of construction is solely determined by the group $\Pi_{\left(s_{1}, s_{2}, \cdots, s_{p}\right)}$ described above. 
Let $Q \subset \mathbb{R}^{4} \rtimes \mathrm{SO}(4)$ be a crystallographic group

$$
\begin{aligned}
Q & =\left\langle\left(\mathbf{v}_{1}, I\right),\left(\mathbf{v}_{2}, I\right),\left(\mathbf{v}_{3}, I\right),\left(\mathbf{v}_{4}, I\right),\left(\mathbf{a}_{1}, A_{1}\right),\left(\mathbf{a}_{2}, A_{2}\right), \cdots,\left(\mathbf{a}_{p}, A_{p}\right)\right\rangle \\
& =\left\langle\mathrm{t}_{1}, \mathrm{t}_{2}, \mathrm{t}_{3}, \mathrm{t}_{4}, \bar{\alpha}_{1}, \bar{\alpha}_{2}, \cdots, \bar{\alpha}_{p}\right\rangle .
\end{aligned}
$$

We assume that the holonomy group $\Phi=\left\langle A_{1}, A_{2}, \cdots, A_{p}\right\rangle$ satisfies

$$
\left\langle A_{1}\right\rangle \triangleleft\left\langle A_{1}, A_{2}\right\rangle \triangleleft \cdots \triangleleft\left\langle A_{1}, A_{2}, \cdots, A_{p}\right\rangle=\Phi .
$$

For $s_{1}, s_{2}, \cdots, s_{p} \in \mathbb{R}^{3}$, denote

$$
\begin{gathered}
t_{1}=\left(0, \mathbf{v}_{1}, I\right), \quad t_{2}=\left(0, \mathbf{v}_{2}, I\right), \quad t_{3}=\left(0, \mathbf{v}_{3}, I\right), \quad t_{4}=\left(0, \mathbf{v}_{4}, I\right), \\
\alpha_{1}=\left(s_{1}, \mathbf{a}_{1}, A_{1}\right), \quad \alpha_{2}=\left(s_{2}, \mathbf{a}_{2}, A_{1}\right), \quad \cdots, \quad \alpha_{p}=\left(s_{p}, \mathbf{a}_{p}, A_{p}\right) .
\end{gathered}
$$

Let $\Pi$ be the group generated by $t_{1}, t_{2}, t_{3}, t_{4}, \alpha_{1}, \alpha_{2}, \cdots, \alpha_{p}$. That is,

$$
\Pi=\Pi_{\left(s_{1}, s_{2}, \cdots, s_{p}\right)}=\left\langle t_{1}, t_{2}, t_{3}, t_{4}, \alpha_{1}, \alpha_{2}, \cdots, \alpha_{p}\right\rangle .
$$

Proposition 6.4 (Description of $\Pi \cap \mathbb{R}^{3}$ ). Every element of $\Pi$ can be written as

$$
t_{1}^{n_{1}} t_{2}^{n_{2}} t_{3}^{n_{3}} t_{4}^{n_{4}} \alpha_{1}^{q_{1}} \alpha_{2}^{q_{2}} \cdots \alpha_{p}^{q_{p}}
$$

(not uniquely). A generating set for $Z=\Pi \cap \mathbb{R}^{3}$ (the pure translations of the group $\Pi$ in the central direction $\mathbb{R}^{3}$ ) is obtained by:

(1) Take $\left[t_{i}, t_{j}\right]$ for all $i, j=1, \cdots, 4$;

(2) Let $\omega=\alpha_{1}^{q_{1}} \alpha_{2}^{q_{2}} \cdots \alpha_{p}^{q_{p}}$. If $\omega^{r}=(w, V, I)$ for some $r>0$, then write $V$ as $V=n_{1} \mathbf{v}_{1}+n_{2} \mathbf{v}_{2}+n_{3} \mathbf{v}_{3}+n_{4} \mathbf{v}_{4}$, and take $t_{1}^{-n_{1}} t_{2}^{-n_{2}} t_{3}^{-n_{3}} t_{4}^{-n_{4}} \omega^{r}$.

Proof. Observe that

$$
\begin{aligned}
\left\langle\mathrm{t}_{1}, \mathrm{t}_{2}, \mathrm{t}_{3}, \mathrm{t}_{4}\right\rangle & \triangleleft\left\langle\mathrm{t}_{1}, \mathrm{t}_{2}, \mathrm{t}_{3}, \mathrm{t}_{4}, \bar{\alpha}_{1}\right\rangle \triangleleft\left\langle\mathrm{t}_{1}, \mathrm{t}_{2}, \mathrm{t}_{3}, \mathrm{t}_{4}, \bar{\alpha}_{1}, \bar{\alpha}_{2}\right\rangle \triangleleft \cdots \\
& \triangleleft\left\langle\mathrm{t}_{1}, \mathrm{t}_{2}, \mathrm{t}_{3}, \mathrm{t}_{4}, \bar{\alpha}_{1}, \cdots, \bar{\alpha}_{p-1}\right\rangle \triangleleft\left\langle\mathrm{t}_{1}, \mathrm{t}_{2}, \mathrm{t}_{3}, \mathrm{t}_{4}, \bar{\alpha}_{1}, \cdots, \bar{\alpha}_{p-1}, \bar{\alpha}_{p}\right\rangle,
\end{aligned}
$$

which implies that every element of $\Pi$ can be written as

$$
\omega=t_{1}^{n_{1}} t_{2}^{n_{2}} t_{3}^{n_{3}} t_{4}^{n_{4}} \alpha_{1}^{q_{1}} \alpha_{2}^{q_{2}} \cdots \alpha_{p}^{q_{p}}
$$

with $n_{i} \in \mathbb{Z}$, and $0 \leq q_{1}<$ order of $A_{1}$, etc.

Clearly the elements in (1) and (2) represent the identity element in the quotient group $Q$. Conversely, an arbitrary element of $\Gamma$ (the lattice of $\mathfrak{N}_{2}$, see the diagram (4-1) ) is a product of $t_{1}^{n_{1}} t_{2}^{n_{2}} t_{3}^{n_{3}} t_{4}^{n_{4}}$ and a power of $\alpha_{1}^{q_{1}} \alpha_{2}^{q_{2}} \cdots \alpha_{p}^{q_{p}}$ whose matrix part is the identity. An element of $\Gamma$ lies in $Z$ if and only if the 4-dimensional vector component is zero. It may not be true that $(0, V, I) \in \Gamma$, but we know $\left(0, \mathbf{v}_{i}, I\right) \in \Gamma$ for $i=1,2,3,4$. We express $V$ in terms of $\mathbf{v}_{i}$ 's, and multiply such $t_{i}=\left(0, \mathbf{v}_{i}, I\right)$ 's in $\Gamma$ to kill $V$. Thus the elements in (1) and (2) form a complete set of generators for $Z$.

We now can take care of the troublesome case: Let $Q$ be an affine 4-dimensional crystallographic group given in 33/08, 33/09 and 33/10 (holonomy group of order 96). The following matrix $P$ will be used to conjugate the given abstract crystallographic group $Q$ (which is embedded into $\mathbb{R}^{4} \rtimes \mathrm{GL}(4)$ ) into $\mathbb{R}^{4} \rtimes \mathrm{SO}(4)$ :

$$
P=\left[\begin{array}{cccc}
1 & 0 & 0 & 0 \\
0 & 1 & 0 & 0 \\
0 & 0 & 1 & 0 \\
\frac{1}{2} & \frac{1}{2} & \frac{1}{2} & \frac{1}{2}
\end{array}\right]
$$


That is, $(\mathbf{0}, P) \in \mathbb{R}^{4} \rtimes \mathrm{GL}(4, \mathbb{R})$ conjugates $Q$ into a genuine crystallographic group. If $S$ is a symmetric matrix which commutes with every element of the holonomy matrices of $(\mathbf{0}, P) Q(\mathbf{0}, P)^{-1}$, it is easy to see that $S=\lambda I$ for some $\lambda \neq 0$. Therefore the condition in Proposition 6.2 is satisfied. Denote

$$
R_{0}=\mu(\mathbf{0}, P)(Q), \quad R_{1}=\mu(\mathbf{0}, \sigma P)(Q),
$$

and denote their holonomy groups by $\Phi_{0}$ and $\Phi_{1}$. We need to check only two specific embeddings $R_{0}$ and $R_{1}=(\mathbf{0}, \sigma) R_{0}(\mathbf{0}, \sigma)^{-1}$.

Let $R=R_{0}$ or $R_{1}$. Write

$$
R=\left\langle\left(\mathbf{v}_{1}, I\right),\left(\mathbf{v}_{2}, I\right),\left(\mathbf{v}_{3}, I\right),\left(\mathbf{v}_{4}, I\right),(\mathbf{a}, A),(\mathbf{b}, B),(\mathbf{c}, C),(\mathbf{d}, D)\right\rangle,
$$

and denote, for some $s, t, u, v \in \mathbb{R}^{3}$,

$$
\begin{array}{ccll}
t_{1}=\left(0, \mathbf{v}_{1}, I\right), & t_{2}=\left(0, \mathbf{v}_{2}, I\right), & t_{3}=\left(0, \mathbf{v}_{3}, I\right), & t_{4}=\left(0, \mathbf{v}_{4}, I\right), \\
\alpha=(s, \mathbf{a}, A), & \beta=(t, \mathbf{b}, B), & \gamma=(u, \mathbf{c}, C), & \delta=(v, \mathbf{d}, D) .
\end{array}
$$

By Proposition 6.3 there is no AB-group from $R$, if and only if for any $s, t, u, v \in \mathbb{R}^{3}$, the group $\Pi$ generated by $t_{1}, t_{2}, t_{3}, t_{4}, \alpha, \beta, \gamma, \delta$,

$$
\Pi_{(s, t, u, v)}=\left\langle t_{1}, t_{2}, t_{3}, t_{4}, \alpha, \beta, \gamma, \delta\right\rangle,
$$

has a non-trivial torsion element. Now let $Z=\Pi \cap \mathbb{R}^{3}$. Then there is no AB-group from $R$ if and only if the following holds: For any $s, t, u, v \in \mathbb{R}^{3}$, there exists a non-trivial torsion element of the form

$$
z t_{1}^{n_{1}} t_{2}^{n_{2}} t_{3}^{n_{3}} t_{4}^{n_{4}} \alpha^{p} \beta^{q} \gamma^{r} \delta^{o}
$$

where $z \in Z$ and $n_{i}, p, q, r, o, k \in \mathbb{Z}$ with $0 \leq p<$ order of $A$, etc.

Proposition 6.5. There is no AB-group $\Pi \subset \mathfrak{N}_{2} \rtimes \operatorname{Aut}\left(\mathfrak{N}_{2}\right)$ constructed from $Q$ given in 33/08 (holonomy group of order 96 ).

Proof. We work with the group $\Pi=\Pi_{(s, t, u, v)}$ in $(6-3)$ which is to be constructed from $R=R_{0}$ or $R_{1}$. We shall show the following: For any $s, t, u, v \in \mathbb{R}^{3}$, there exist $z \in Z$, and $n_{i}, p, q, r, o, k \in \mathbb{Z}$ with $0 \leq p<2,0 \leq q<4,0 \leq r<2,0 \leq o<12$ such that

$$
z t_{1}^{n_{1}} t_{2}^{n_{2}} t_{3}^{n_{3}} t_{4}^{n_{4}} \alpha^{p} \beta^{q} \gamma^{r} \delta^{o}
$$

is a non-trivial torsion element.

Case $R=R_{0}$. The finite subgroup $Q_{0}=\left\langle(b, B),(d, D)^{3}\right\rangle$ in $R_{0}$ forms a quaternion group (non-cyclic group of order 8) with $Q_{1}=Q_{0}$. This contradicts Lemma 5.2

Case $R=R_{1}$. Our $R_{1}$ has a representation

$$
R_{1}=\left\langle\left(\mathbf{v}_{1}, I\right),\left(\mathbf{v}_{2}, I\right),\left(\mathbf{v}_{3}, I\right),\left(\mathbf{v}_{4}, I\right),(\mathbf{a}, A),(\mathbf{b}, B),(\mathbf{c}, C),(\mathbf{d}, D)\right\rangle,
$$

where

$$
\mathbf{v}_{1}=\left[\begin{array}{r}
-1 \\
0 \\
0 \\
-1
\end{array}\right], \quad \mathbf{v}_{2}=\left[\begin{array}{r}
0 \\
1 \\
0 \\
-1
\end{array}\right], \quad \mathbf{v}_{3}=\left[\begin{array}{r}
0 \\
0 \\
1 \\
-1
\end{array}\right], \quad \mathbf{v}_{4}=\left[\begin{array}{l}
0 \\
0 \\
0 \\
2
\end{array}\right]
$$




$$
\begin{aligned}
(\mathbf{a}, A) & =\left(\left[\begin{array}{l}
0 \\
0 \\
0 \\
1
\end{array}\right], \quad\left[\begin{array}{rrrr}
1 & 0 & 0 & 0 \\
0 & -1 & 0 & 0 \\
0 & 0 & -1 & 0 \\
0 & 0 & 0 & 1
\end{array}\right]\right), \\
(\mathbf{b}, B) & =\left(\left[\begin{array}{l}
0 \\
0 \\
0 \\
0
\end{array}\right], \quad\left[\begin{array}{rrrr}
0 & 0 & 0 & -1 \\
0 & 0 & 1 & 0 \\
0 & -1 & 0 & 0 \\
1 & 0 & 0 & 0
\end{array}\right]\right), \\
(\mathbf{c}, C) & =\left(\frac{1}{2}\left[\begin{array}{r}
-1 \\
1 \\
1 \\
-1
\end{array}\right], \quad\left[\begin{array}{rrrr}
0 & 0 & 1 & 0 \\
0 & 0 & 0 & 1 \\
1 & 0 & 0 & 0 \\
0 & 1 & 0 & 0
\end{array}\right]\right) \\
(\mathbf{d}, D) & =\left(\left[\begin{array}{l}
0 \\
0 \\
0 \\
0
\end{array}\right], \quad \frac{1}{2}\left[\begin{array}{rrrr}
-1 & -1 & 1 \\
-1 & 1 & -1 \\
-1 & -1 & -1 \\
1 & -1 & -1 & -1 \\
1
\end{array}\right]\right) .
\end{aligned}
$$

For $s, t, u, v \in \mathbb{R}^{3}$, the group $\Pi=\Pi_{(s, t, u, v)}$ as in (6-3) has a generating set

$$
\begin{array}{llll}
t_{1}=\left(0, \mathbf{v}_{1}, I\right), & t_{2}=\left(0, \mathbf{v}_{2}, I\right), & t_{3}=\left(0, \mathbf{v}_{3}, I\right), & t_{4}=\left(0, \mathbf{v}_{4}, I\right), \\
\alpha=(s, \mathbf{a}, A), & \beta=(t, \mathbf{b}, B), & \gamma=(u, \mathbf{c}, C), & \delta=(v, \mathbf{d}, D) .
\end{array}
$$

Then

$$
Z=\Pi \cap \mathbb{R}^{3}
$$

is a lattice of $\mathbb{R}^{3}$. Let

$$
z=\beta^{-4}=\left(\left[\begin{array}{c}
0 \\
0 \\
-4 \mathrm{t}_{3}
\end{array}\right], \mathbf{0}, I\right) .
$$

Then $z$ is an element of $Z$, and

$$
z\left(\beta \delta^{2}\right)^{4}=\beta^{-4}\left(\beta \delta^{2}\right)^{4}=\left(0, \mathbf{0},\left(B D^{2}\right)^{4}\right)
$$

is a torsion element of order 3 (for any choice of $s, t, u, v$ ).

Corollary 6.6. There is no AB-group $\Pi \subset \mathfrak{N}_{2} \rtimes \operatorname{Aut}\left(\mathfrak{N}_{2}\right)$ constructed from $Q$ given in 33/12 (holonomy group of order 192).

Proof. Consider the subgroup $S$ of $Q$ generated by the following elements:

$$
\left(\mathbf{e}_{1}, I\right),\left(\mathbf{e}_{2}, I\right),\left(\mathbf{e}_{3}, I\right),\left(\mathbf{e}_{4}, I\right),(\mathbf{a}, A),(\mathbf{b}, B),(\mathbf{c}, C),(\mathbf{d}, D)
$$

[we removed $(e, E)]$. Then $S$ will be a 4 -dimensional crystallographic group, and its holonomy group is generated by $A, B, C, D$. Since the holonomy group of $Q$ has order 192, the holonomy group of $S$ must have order 96, and is normal in $Q$. If we prove that there is no AB-group constructed from any 4-dimensional crystallographic group with holonomy group of order 96 (in particular, there is no AB-group constructed from our $S$ ), then we will have proved that there is no AB-group constructed from $Q$.

In fact we can see that our $S$ is one in the list already: The subgroup $S$ of $Q$ given above is actually isomorphic to the group $Q^{\prime}$ given in 33/08 with generators

$$
\left(\mathbf{e}_{1}, I\right),\left(\mathbf{e}_{2}, I\right),\left(\mathbf{e}_{3}, I\right),\left(\mathbf{e}_{4}, I\right),\left(\mathbf{a}^{\prime}, A^{\prime}\right),\left(\mathbf{b}^{\prime}, B^{\prime}\right),\left(\mathbf{c}^{\prime}, C^{\prime}\right),\left(\mathbf{d}^{\prime}, D^{\prime}\right) .
$$


Take

$$
\mathbf{v}=\left[\begin{array}{r}
0 \\
0 \\
-\frac{1}{2} \\
-\frac{1}{2}
\end{array}\right]
$$

Then

$$
\begin{aligned}
& \mu(\mathbf{v}, I)(\mathbf{a}, A)=\left(\mathbf{e}_{3}, I\right)\left(\mathbf{a}^{\prime}, A^{\prime}\right), \\
& \mu(\mathbf{v}, I)(\mathbf{b}, B)=\left(\mathbf{e}_{3}, I\right)\left(\mathbf{b}^{\prime}, B^{\prime}\right), \\
& \mu(\mathbf{v}, I)(\mathbf{c}, C)=\left(\mathbf{e}_{2}, I\right)^{-1}\left(\mathbf{c}^{\prime}, C^{\prime}\right), \\
& \mu(\mathbf{v}, I)(\mathbf{d}, D)=\left(\mathbf{e}_{2}, I\right)\left(\mathbf{e}_{4}, I\right)\left(\mathbf{b}^{\prime}, B^{\prime}\right)\left(\mathbf{d}^{\prime}, D^{\prime}\right)^{-1} .
\end{aligned}
$$

This shows that $\mu(\mathbf{v}, I): S \rightarrow Q^{\prime}$ is an isomorphism.

Proposition 6.7. There is no AB-group $\Pi \subset \mathfrak{N}_{2} \rtimes \operatorname{Aut}\left(\mathfrak{N}_{2}\right)$ constructed from $Q$ given in 33/10 (holonomy group of order 96 ).

Proof. We proceed as in the case of $\mathbf{3 3 / 0 8}$. Let $Q$ be the group given in $\mathbf{3 3 / 1 0}$. Consider two embeddings $R_{0}$ and $R_{1}$ of $Q$ into $\mathbb{R}^{4} \rtimes \mathrm{SO}(4)$ by conjugating by $(\mathbf{0}, P)$ and $(\mathbf{0}, \sigma P)$, respectively. Let

$$
R_{0}=\mu(\mathbf{0}, P)(Q), \quad R_{1}=\mu(\mathbf{0}, \sigma P)(Q),
$$

as before.

Case $R=R_{0}$. Our $R_{0}$ has a representation

$$
R_{0}=\left\langle\left(\mathbf{v}_{1}, I\right),\left(\mathbf{v}_{2}, I\right),\left(\mathbf{v}_{3}, I\right),\left(\mathbf{v}_{4}, I\right),(\mathbf{a}, A),(\mathbf{b}, B),(\mathbf{c}, C),(\mathbf{d}, D)\right\rangle,
$$

where

$$
\begin{aligned}
& \mathbf{v}_{1}=\left[\begin{array}{r}
1 \\
0 \\
0 \\
-1
\end{array}\right], \quad \mathbf{v}_{2}=\left[\begin{array}{r}
0 \\
1 \\
0 \\
-1
\end{array}\right], \quad \mathbf{v}_{3}=\left[\begin{array}{r}
0 \\
0 \\
1 \\
-1
\end{array}\right], \quad \mathbf{v}_{4}=\left[\begin{array}{l}
0 \\
0 \\
0 \\
2
\end{array}\right] \text {, } \\
& (\mathbf{a}, A)=\left(\left[\begin{array}{l}
0 \\
0 \\
0 \\
0
\end{array}\right], \quad\left[\begin{array}{rrrr}
0 & 0 & 0 & 1 \\
0 & 0 & 1 & 0 \\
0 & -1 & 0 & 0 \\
-1 & 0 & 0 & 1
\end{array}\right]\right), \\
& (\mathbf{b}, B)=\left(\left[\begin{array}{l}
0 \\
0 \\
0 \\
1
\end{array}\right], \quad\left[\begin{array}{rrrr}
0 & 0 & -1 & 0 \\
0 & 0 & 0 & 1 \\
1 & 0 & 0 & 0 \\
0 & -1 & 0 & 0
\end{array}\right]\right) \\
& (\mathbf{c}, C)=\left(\frac{1}{2}\left[\begin{array}{r}
1 \\
1 \\
1 \\
-3
\end{array}\right], \quad\left[\begin{array}{rrrr}
-1 & -1 & -1 & -1 \\
1 & -1 & -1 & 1 \\
1 & 1 & -1 & -1 \\
1 & -1 & 1 & -1
\end{array}\right]\right) \\
& (\mathbf{d}, D)=\left(\frac{1}{2}\left[\begin{array}{r}
1 \\
1 \\
1 \\
-3
\end{array}\right], \quad\left[\begin{array}{rrrr}
0 & 0 & 0 & 1 \\
0 & 1 & 0 & 0 \\
0 & 0 & 1 & 0 \\
-1 & 0 & 0 & 0
\end{array}\right]\right) \text {. }
\end{aligned}
$$


For $s, t, u, v \in \mathbb{R}^{3}$, the group $\Pi=\Pi_{(s, t, u, v)}$ as in (6-3) has a generating set

$$
\begin{array}{llll}
t_{1}=\left(0, \mathbf{v}_{1}, I\right), & t_{2}=\left(0, \mathbf{v}_{2}, I\right), & t_{3}=\left(0, \mathbf{v}_{3}, I\right), & t_{4}=\left(0, \mathbf{v}_{4}, I\right), \\
\alpha=(s, \mathbf{a}, A), & \beta=(t, \mathbf{b}, B), & \gamma=(u, \mathbf{c}, C), & \delta=(v, \mathbf{d}, D) .
\end{array}
$$

Then

$$
Z=\Pi \cap \mathbb{R}^{3}
$$

is a lattice of $\mathbb{R}^{3}$. Let

$$
z=\left(\left[t_{1}, t_{4}\right]^{3}(\gamma \delta)^{8}\right)^{-1}=\left(\left[\begin{array}{c}
0 \\
0 \\
76-8 \mathrm{u}_{3}-8 \mathrm{v}_{3}
\end{array}\right], \mathbf{0}, I\right) .
$$

Then $z$ is an element of $Z$, and

$$
z \cdot\left(\gamma^{2} \delta^{2}\right)^{4}=\left(\left[t_{1}, t_{2}\right]^{3}(\gamma \delta)^{8}\right)^{-1} \cdot\left(\gamma^{2} \delta^{2}\right)^{4}
$$

is a torsion element of order 3 (for any choice of $s, t, u, v$ ).

Case $R=R_{1}$. The finite subgroup

$$
Q_{0}=\left\langle(a, A)(b, B)^{3}(c, C)(d, D), t_{2}^{-1} t_{4}^{-1}(b, B)^{2}(c, C)\right\rangle
$$

in $R_{1}$ forms a group of order 24 with $Q_{0}=\mathbb{Z}_{4}$, and $Q_{2} \cong \mathbb{Z}_{3} \rtimes \mathbb{Z}_{2}$ a non-cyclic group of order 6 . This contradicts Lemma 5.2

Proposition 6.8. There is no AB-group $\Pi \subset \mathfrak{N}_{2} \rtimes \operatorname{Aut}\left(\mathfrak{N}_{2}\right)$ constructed from $Q$ given in 33/09 (holonomy group of order 96 ).

Proof. Let $Q$ be the group given in 33/09. Consider two embeddings $R_{0}$ and $R_{1}$ of $Q$ into $\mathbb{R}^{4} \rtimes \mathrm{SO}(4)$ by conjugating by $(\mathbf{0}, P)$ and $(\mathbf{0}, \sigma P)$, respectively. Let

$$
R_{0}=\mu(\mathbf{0}, P)(Q), \quad R_{1}=\mu(\mathbf{0}, \sigma P)(Q)
$$

as before.

Case $R=R_{0}$. Our $R_{0}$ has a representation

$$
R_{1}=\left\langle\left(\mathbf{v}_{1}, I\right),\left(\mathbf{v}_{2}, I\right),\left(\mathbf{v}_{3}, I\right),\left(\mathbf{v}_{4}, I\right),(\mathbf{a}, A),(\mathbf{b}, B),(\mathbf{c}, C),(\mathbf{d}, D)\right\rangle,
$$

where

$$
\mathbf{v}_{1}=\left[\begin{array}{r}
1 \\
0 \\
0 \\
-1
\end{array}\right], \quad \mathbf{v}_{2}=\left[\begin{array}{r}
0 \\
1 \\
0 \\
-1
\end{array}\right], \quad \mathbf{v}_{3}=\left[\begin{array}{r}
0 \\
0 \\
1 \\
-1
\end{array}\right], \quad \mathbf{v}_{4}=\left[\begin{array}{l}
0 \\
0 \\
0 \\
2
\end{array}\right]
$$




$$
\begin{aligned}
(\mathbf{a}, A) & =\left(\left[\begin{array}{l}
0 \\
0 \\
0 \\
0
\end{array}\right], \quad\left[\begin{array}{rrrr}
0 & 0 & 0 & 1 \\
0 & 0 & 1 & 0 \\
0 & -1 & 0 & 0 \\
-1 & 0 & 0 & 0
\end{array}\right]\right), \\
(\mathbf{b}, B) & =\left(\left[\begin{array}{l}
0 \\
0 \\
0 \\
1
\end{array}\right], \quad\left[\begin{array}{rrrr}
0 & 0 & -1 & 0 \\
0 & 0 & 0 & 1 \\
1 & 0 & 0 & 0 \\
0 & -1 & 0 & 0
\end{array}\right]\right) \\
(\mathbf{c}, C) & =\left(\frac{1}{2}\left[\begin{array}{l}
0 \\
0 \\
1 \\
1
\end{array}\right], \quad \frac{1}{2}\left[\begin{array}{rrrr}
-1 & -1 & 1 & -1 \\
1 & -1 & 1 & 1 \\
1 & 1 & 1 & -1
\end{array}\right]\right) \\
(\mathbf{d}, D) & =\left(\left[\begin{array}{l}
0 \\
0 \\
0 \\
0
\end{array}\right], \quad\left[\begin{array}{rrrr}
1 & 0 & 0 & 0 \\
0 & 0 & 1 & 0 \\
0 & 1 & 0 & 0 \\
0 & 0 & 0 & -1
\end{array}\right]\right)
\end{aligned}
$$

For $s, t, u, v \in \mathbb{R}^{3}$, the group $\Pi=\Pi_{(s, t, u, v)}$ as in (6-3) has a generating set

$$
\begin{array}{llll}
t_{1}=\left(0, \mathbf{v}_{1}, I\right), & t_{2}=\left(0, \mathbf{v}_{2}, I\right), & t_{3}=\left(0, \mathbf{v}_{3}, I\right), & t_{4}=\left(0, \mathbf{v}_{4}, I\right), \\
\alpha=(s, \mathbf{a}, A), & \beta=(t, \mathbf{b}, B), & \gamma=(u, \mathbf{c}, C), & \delta=(v, \mathbf{d}, D) .
\end{array}
$$

Then

$$
Z=\Pi \cap \mathbb{R}^{3}
$$

is a lattice of $\mathbb{R}^{3}$, and one can show that $Z$ is generated by the following 12 elements (using row notation instead of column notation):

$$
\begin{aligned}
& w_{1}=\left(-1+\mathrm{s}_{1}, 1+\mathrm{s}_{2}, \mathrm{~s}_{3}\right), \\
& w_{2}=\left(-1+\mathrm{t}_{1}, \mathrm{t}_{2},-\mathrm{t}_{3}\right) \\
& w_{3}=\left(\mathrm{v}_{1}+\mathrm{v}_{2}, \mathrm{v}_{1}+\mathrm{v}_{2}, 0\right) \\
& w_{4}=\left(-\mathrm{t}_{2}, 1-\mathrm{t}_{1},-\mathrm{t}_{3}\right) \\
& w_{5}=\left(-1+\mathrm{t}_{1}, \mathrm{t}_{2}, \mathrm{t}_{3}\right), \\
& w_{6}=\left(0,0,2 \mathrm{~s}_{3}\right) \\
& w_{7}=\left(-\mathrm{s}_{1}-\mathrm{s}_{2},-\mathrm{s}_{1}-\mathrm{s}_{2}, 0\right) \\
& w_{8}=\left(\mathrm{s}_{1}-\mathrm{s}_{2},-\mathrm{s}_{1}+\mathrm{s}_{2},-2\right) \\
& w_{9}=\left(1-\mathrm{s}_{1}+\mathrm{s}_{2}+\mathrm{t}_{1}+\mathrm{t}_{2}, 1+\mathrm{s}_{1}-\mathrm{s}_{2}+\mathrm{t}_{1}+\mathrm{t}_{2}, 0\right), \\
& w_{10}=\left(-\mathrm{s}_{1}+\mathrm{s}_{2}, \mathrm{~s}_{1}-\mathrm{s}_{2},-2\right) \\
& w_{11}=\left(\mathrm{s}_{1}-\mathrm{s}_{2}+\mathrm{u}_{1}-\mathrm{u}_{2}-\mathrm{v}_{1}+\mathrm{v}_{2},-\mathrm{s}_{1}+\mathrm{s}_{2}-\mathrm{u}_{1}+\mathrm{u}_{2}+\mathrm{v}_{1}-\mathrm{v}_{2}, 0\right), \\
& w_{12}=\left(0,0,1+6 \mathrm{u}_{3}\right)
\end{aligned}
$$

[This, as well as other parts of calculations, was done by the program MATHEMATICA [10] and hand-checked.]

We note that

$$
\begin{aligned}
& (0,0,4)=-w_{8}-w_{10} \\
& (4,4,0)=-2 w_{2}+2 w_{4}+w_{8}+2 w_{9}-w_{10} \\
& (2,2,2)=-w_{2}+w_{4}+w_{9}-w_{10}
\end{aligned}
$$

are elements of $Z$.

Let $\left(2 x_{0}, 2 x_{0}, 0\right) \in Z$ be a generator of the subgroup of $Z$ consisting of the elements of the form $(x, x, 0)$. Since $(4,4,0) \in Z, x_{0}$ is a rational number. Note 
also that $w_{3}=\left(v_{1}+v_{2}, v_{1}+v_{2}, 0\right) \in Z$. Therefore, there exist integers $m$ and $p$ such that

$$
v_{1}+v_{2}=m \cdot 2 x_{0}, \quad 4=p \cdot 2 x_{0} .
$$

(1) Suppose $p=2 \ell$, even. Then $4=2 \ell \cdot 2 x_{0}$ shows $2=\ell \cdot 2 x_{0}$, and hence $(2,2,0)=\ell\left(2 x_{0}, 2 x_{0}, 0\right) \in Z$. Since $(2,2,2) \in Z$, we have $(0,0,2) \in Z$. Consider $z=-w_{12}+5(0,0,2) \in Z$. It is easy to see that $z \gamma^{6} \in \Pi$ is a torsion element of order 2 .

(2) Suppose $p=2 \ell+1$, odd. Then $4=(2 \ell+1) \cdot 2 x_{0}$ yields

$$
\begin{aligned}
(2,2,0) & =(2 \ell+1)\left(x_{0}, x_{0}, 0\right) \\
& =\ell\left(2 x_{0}, 2 x_{0}, 0\right)+\left(x_{0}, x_{0}, 0\right) .
\end{aligned}
$$

Therefore,

$$
(2,2,2)=\ell\left(2 x_{0}, 2 x_{0}, 0\right)+\left(x_{0}, x_{0}, 2\right)
$$

which shows $\left(x_{0}, x_{0}, 2\right) \in Z$. Let

$$
z=-m\left(x_{0}, x_{0}, 2\right) \in Z \text {. }
$$

Then it is easy to see that $z \delta \in \Pi$ is a torsion element of order 2 .

Case $R=R_{1}$. The finite subgroup $Q_{0}=\left\langle(c, C)^{4},(a, A)(d, D)\right\rangle$ in $R_{1}$ forms a non-commutative holonomy group of order 6 . This contradicts Lemma 5.2 .

\section{A CONStruction of AN AB-GRoup With holonomy ORDER 48}

Theorem 7.1 (Existence). There exists an almost Bieberbach group $\Pi \subset \mathfrak{N}_{2} \rtimes$ $\operatorname{Aut}\left(\mathfrak{N}_{2}\right)$ whose holonomy group has order 48.

Proof. Consider the 4-dimensional crystallographic group $Q$ (Case 33/05/01/003) generated by the following elements:

$$
\left(\mathbf{e}_{1}, I\right), \quad\left(\mathbf{e}_{2}, I\right), \quad\left(\mathbf{e}_{3}, I\right), \quad\left(\mathbf{e}_{4}, I\right), \quad(\mathbf{a}, A), \quad(\mathbf{b}, B), \quad(\mathbf{c}, C),
$$

where $I$ is the identity matrix, $\mathbf{e}_{i}$ is the unit vector with 1 in the $i$ th coordinate, and

$$
\begin{aligned}
& (\mathbf{a}, A)=\left(\frac{1}{2}\left[\begin{array}{l}
1 \\
1 \\
1 \\
1
\end{array}\right], \quad\left[\begin{array}{rrrr}
-1 & -1 & -1 & 2 \\
0 & 0 & 1 & 0 \\
0 & -1 & 0 & 0 \\
-1 & -1 & 0 & 1
\end{array}\right]\right), \\
& (\mathbf{b}, B)=\left(\frac{1}{2}\left[\begin{array}{l}
1 \\
1 \\
1 \\
0
\end{array}\right], \quad\left[\begin{array}{rrrr}
0 & 0 & -1 & 0 \\
-1 & -1 & -1 & 2 \\
1 & 0 & 0 & 0 \\
0 & -1 & -1 & 1
\end{array}\right]\right), \\
& (\mathbf{c}, C)=\left(\left[\begin{array}{l}
0 \\
0 \\
0 \\
0
\end{array}\right], \quad\left[\begin{array}{rrrr}
0 & 0 & 0 & -1 \\
0 & 1 & 1 & -1 \\
-1 & -1 & 0 & 1 \\
0 & 0 & 1 & -1
\end{array}\right]\right) .
\end{aligned}
$$

Note that

$$
A^{4}=I, \quad B^{2}=A^{2}, \quad C^{6}=A^{2}, \quad[A, B]=A^{2}, \quad[A, C]=B A^{2}, \quad[B, C]=B A^{3}
$$

so that $Q$ has the holonomy group of order 48 . 
Now we conjugate this group by the matrix

$$
P=\left[\begin{array}{cccc}
1 & 0 & 0 & 0 \\
0 & 1 & 0 & 0 \\
0 & 0 & 1 & 0 \\
\frac{1}{2} & \frac{1}{2} & \frac{1}{2} & \frac{1}{2}
\end{array}\right]
$$

Then the new group has the following representation inside $\mathbb{R}^{4} \rtimes \mathrm{SO}(4)$ :

$$
\begin{gathered}
\left(\mathbf{v}_{1}, I\right), \quad\left(\mathbf{v}_{2}, I\right), \quad\left(\mathbf{v}_{3}, I\right), \quad\left(\mathbf{v}_{4}, I\right), \\
(\mathbf{a}, A)=\left(\frac{1}{2}\left[\begin{array}{r}
1 \\
1 \\
1 \\
-1
\end{array}\right], \quad\left[\begin{array}{rrrr}
0 & 0 & 0 & 1 \\
0 & 0 & 1 & 0 \\
0 & -1 & 0 & 0 \\
-1 & 0 & 0 & 0
\end{array}\right]\right), \\
(\mathbf{b}, B)=\left(\frac{1}{2}\left[\begin{array}{r}
1 \\
1 \\
1 \\
-3
\end{array}\right], \quad\left[\begin{array}{rrrr}
0 & 0 & -1 & 0 \\
0 & 0 & 0 & 1 \\
1 & 0 & 0 & 0 \\
0 & -1 & 0 & 0
\end{array}\right]\right), \\
(\mathbf{c}, C)=\left(\left[\begin{array}{l}
0 \\
0 \\
0 \\
0
\end{array}\right], \frac{1}{2}\left[\begin{array}{rrrr}
-1 & -1 & -1 & -1 \\
-1 & 1 & 1 & -1 \\
-1 & -1 & 1 & 1 \\
1 & -1 & 1 & -1
\end{array}\right]\right),
\end{gathered}
$$

where

$$
\mathbf{v}_{1}=\left[\begin{array}{r}
1 \\
0 \\
0 \\
-1
\end{array}\right], \quad \mathbf{v}_{2}=\left[\begin{array}{r}
0 \\
1 \\
0 \\
-1
\end{array}\right], \quad \mathbf{v}_{3}=\left[\begin{array}{r}
0 \\
0 \\
1 \\
-1
\end{array}\right], \quad \mathbf{v}_{4}=\left[\begin{array}{l}
0 \\
0 \\
0 \\
2
\end{array}\right]
$$

Next we consider the subgroup $\Pi$ of $\mathbb{R}^{3} \tilde{\times} \mathbb{R}^{4} \rtimes \mathrm{SO}(4)$ generated by

$$
\begin{gathered}
t_{1}=\left(0, \mathbf{v}_{1}, I\right), \quad t_{2}=\left(0, \mathbf{v}_{2}, I\right), \quad t_{3}=\left(0, \mathbf{v}_{3}, I\right), \quad t_{4}=\left(0, \mathbf{v}_{4}, I\right), \\
\alpha=\left(\left[\begin{array}{l}
0 \\
0 \\
0
\end{array}\right], \mathbf{a}, A\right), \quad \beta=\left(\left[\begin{array}{l}
0 \\
0 \\
0
\end{array}\right], \mathbf{b}, B\right), \quad \gamma=\left(\left[\begin{array}{r}
-\frac{1}{6} \\
-\frac{1}{6} \\
\frac{1}{6}
\end{array}\right], \mathbf{c}, C\right) .
\end{gathered}
$$

Let $Z=\Pi \cap \mathbb{R}^{3}$. We already have a detailed description of $Z$ in Proposition 6.4 By calculation, we see that $Z$ is actually generated by the vectors

$$
z_{1}=\left[\begin{array}{r}
-1 \\
1 \\
1
\end{array}\right], \quad z_{2}=\left[\begin{array}{r}
1 \\
-1 \\
1
\end{array}\right], \quad z_{3}=\left[\begin{array}{l}
1 \\
1 \\
0
\end{array}\right]
$$

In particular, $Z$ is discrete and hence $\Pi$ is discrete.

Next we claim that $\Pi$ is torsion free. Every element of $\Pi$ can be written as

$$
z t_{1}^{n_{1}} t_{2}^{n_{2}} t_{3}^{n_{3}} t_{4}^{n_{4}} \alpha^{p} \beta^{q} \gamma^{r}
$$

where $z \in Z$, and $n_{1}, n_{2}, n_{3}, p, q, r$ are integers such that $0 \leq p<4,0 \leq q<4$ and $0 \leq r<12$. If $z t_{1}^{n_{1}} t_{2}^{n_{2}} t_{3}^{n_{3}} t_{4}^{n_{4}} \alpha^{p} \beta^{q} \gamma^{r}$ is a torsion element of order $g$, then $\left(A^{p} B^{q} C^{r}\right)^{g}=I$. Thus it suffices to consider all the torsion elements of $\Phi$ whose 
order is a prime dividing 48 . The following shows the list of prime ordered elements of $\Phi$ :

Order 2 elements: $A^{2}, A C^{3}, A B C^{3}, A^{3} C^{3}, A^{2} B C^{3}, A^{3} B C^{3}, B C^{3}$.

Order 3 elements: $A^{3} B C^{2}, A^{3} B C^{4}, A C^{2}, A^{2} C^{2}, A C^{4}, B C^{2}, B C^{4}, C^{4}$.

It can be shown that none of the corresponding 15 equations

$$
\begin{gathered}
\left(z_{1}^{\ell_{1}} z_{2}^{\ell_{2}} z_{3}^{\ell_{3}} t_{1}^{n_{1}} t_{2}^{n_{2}} t_{3}^{n_{3}} t_{4}^{n_{4}} \alpha^{2} \beta^{0} \gamma^{0}\right)^{2}=(0, \mathbf{0}, I), \\
\left(z_{1}^{\ell_{1}} z_{2}^{\ell_{2}} z_{3}^{\ell_{3}} t_{1}^{n_{1}} t_{2}^{n_{2}} t_{3}^{n_{3}} t_{4}^{n_{4}} \alpha^{1} \beta^{0} \gamma^{3}\right)^{2}=(0, \mathbf{0}, I), \\
\left(z_{1}^{\ell_{1}} z_{2}^{\ell_{2}} z_{3}^{\ell_{3}} t_{1}^{n_{1}} t_{2}^{n_{2}} t_{3}^{n_{3}} t_{4}^{n_{4}} \alpha^{1} \beta^{1} \gamma^{3}\right)^{2}=(0, \mathbf{0}, I), \\
\ldots \\
\left(z_{1}^{\ell_{1}} z_{2}^{\ell_{2}} z_{3}^{\ell_{3}} t_{1}^{n_{1}} t_{2}^{n_{2}} t_{3}^{n_{3}} t_{4}^{n_{4}} \alpha^{0} \beta^{0} \gamma^{4}\right)^{3}=(0, \mathbf{0}, I)
\end{gathered}
$$

has any integral solutions for $\ell_{1}, \ell_{2}, \ell_{3}, n_{1}, n_{2}, n_{3}, n_{4}$. This proves that $\Pi$ is indeed torsion free, and thus is an AB-group.

\section{Non-EXistence}

Now a case-by-case study of the table from [1] shows that all 4-dimensional crystallographic groups with holonomy group of order greater than 48 satisfy at least one of the disqualification criteria proved so far. Using notations from [1, we list them below. All the calculations were done using the program MATHEMATICA [10] and hand-checked.

If the holonomy group of $Q$ contains a matrix of determinant -1 , then $Q$ is excluded by Corollary 5.1. Also if $Q$ contains $\left(\mathbb{Z}_{2}\right)^{3}$, then $Q$ is excluded by Lemma 5.3

- $\underline{1152}$ :

33/16: $\operatorname{det}(G)=-1$.

- $\underline{576}$ :

33/14: $\operatorname{det}(F)=-1$,

$33 / 15:\left\langle A, B^{2}, F\right\rangle \cong\left(\mathbb{Z}_{2}\right)^{3}$.

- $\underline{384}$ :

32/21: $\operatorname{det}(F)=-1$.

- $\underline{288}$ :

30/13: $\operatorname{det}(D)=-1$,

33/13: $\left\langle B D^{3}, C, D^{6}\right\rangle \cong\left(\mathbb{Z}_{2}\right)^{3}$.

- $\underline{240}$ :

31/07: $\operatorname{det}(C)=-1$.

- $\underline{192}$ :

32/18: $\operatorname{det}(E)=-1$,

32/19: $\operatorname{det}(F)=-1$,

32/20/01, 03: $\left\langle(a, A)^{2},(b, B),(c, C)\right\rangle \cong\left(\mathbb{Z}_{2}\right)^{3}$,

$32 / 20 / 02:\left\langle(a, A)^{2}(b, B),(a, A)(c, C), t_{2}(b, B)\right\rangle \cong\left(\mathbb{Z}_{2}\right)^{3}$,

33/12: Corollary 6.6. 
- $144:$

23/11, 29/09, 30/11, 30/12: $\operatorname{det}(C)=-1$,

30/10: $\left\langle A, C^{3}, D\right\rangle \cong\left(\mathbb{Z}_{2}\right)^{3}$,

33/11: The non-abelian group $\langle A C, B\rangle$ of order 24 lies completely inside $\Phi_{\ell}$ or $\Phi_{r}$. Apply Corollary [5.6.

- $\underline{128}$ :

$32 / 17: \operatorname{det}(D)=-1$.

- $\underline{120}$ :

31/04, 31/05: $\operatorname{det}(A)=-1$,

31/06: $\left\langle A, B, D^{5}\right\rangle \cong\left(\mathbb{Z}_{2}\right)^{3}$.

- $\underline{96}$ :

20/22: $\operatorname{det}(D)=-1$,

25/11: $\operatorname{det}(E)=-1$,

$32 / 16 / 01,32 / 16 / 03:\left\langle A^{2}, C, D\right\rangle \cong\left(\mathbb{Z}_{2}\right)^{3}$,

$32 / 16 / 02:\left\langle t_{2}^{-1} t_{4}^{-1}(d, D), t_{4}^{-1}(c, D), t_{1} t_{2}^{-1}(a, A)^{2}\right\rangle \cong\left(\mathbb{Z}_{2}\right)^{3}$,

33/08: Proposition 6.5

33/09: Proposition 6.8

33/10: Proposition 6.7

- $\underline{72}$ :

22/11, 23/09, 23/10: $\operatorname{det}(B)=-1$,

23/07, 29/06, 29/08: $\operatorname{det}(C)=-1$,

29/07: $\operatorname{det}(D)=-1$,

23/08, 29/05: $\left\langle A^{3}, B^{3}, C\right\rangle \cong\left(\mathbb{Z}_{2}\right)^{3}$,

30/07: $\langle B, B C\rangle$ generates a non-abelian group of order 72 with the highest period of the elements in $\Phi_{\ell} \cup \Phi_{r}$ 6. Apply Corollary 5.7.

30/08: $\left\langle A B^{4}, B C\right\rangle$ induces a non-commutative holonomy of order 6, Lemma 5.2,

30/09: $\left\langle A B^{4}, C\right\rangle$ induces a non-commutative holonomy of order 6, Lemma 5.2,

33/07: $\left\langle A, C^{2}\right\rangle$ generates a non-abelian group of order 72 with the highest period of the elements in $\Phi_{\ell} \cup \Phi_{r}$ 6. Apply Corollary 5.7.

- $\underline{64}$ :

19/06 (C), 32/13 (D), 32/14 (D), 32/15 (C): $\operatorname{det}=-1$,

32/12/01/002: $\left\langle t_{3}(a, A)^{4}(c, C),(b, B),(c, C)\right\rangle \cong\left(\mathbb{Z}_{2}\right)^{3}$,

32/12/01/003: $\left\langle t_{2}^{-1}(a, A)^{4}(b, B),(b, B),(d, D)\right\rangle \cong\left(\mathbb{Z}_{2}\right)^{3}$,

32/12/01/004: $\left\langle t_{1}(a, A)^{6}(d, D), t_{3}^{-1}(a, A)^{2}(d, D),(b, B)\right\rangle \cong\left(\mathbb{Z}_{2}\right)^{3}$,

32/12/02/002: $\left\langle(a, A)^{4},(b, B)(c, C), t_{2}^{-1}(c, C)\right\rangle \cong\left(\mathbb{Z}_{2}\right)^{3}$,

32/12/02/003: $\left\langle t_{3}^{-1}(a, A)^{2}(c, C), t_{2}^{-1}(a, A)^{6}(c, C)\right.$, $\left.t_{2} t_{3}^{-2}(a, A)^{6}(b, B)(c, C)\right\rangle \cong\left(\mathbb{Z}_{2}\right)^{3}$,

32/12/02/004: Proposition 5.8,

32/12/02/005: $\left\langle t_{2}(a, A)^{6}(d, D), t_{4}^{-1}(a, A)^{2}(d, D), t_{2}(b, B)\right\rangle \cong\left(\mathbb{Z}_{2}\right)^{3}$,

32/12/02/006: $\left\langle t_{1}^{-1} t_{2}^{2}(a, A)^{6}(d, D), t_{2} t_{3}(a, A)^{4}(b, B), t_{2}(b, B)\right\rangle \cong\left(\mathbb{Z}_{2}\right)^{3}$. 
- $\underline{60}$ :

31/03/01: $\left\langle t_{1}^{-1}(d, D)^{3}, t_{1}^{-1} t_{2}^{-1}(b, B)(b, B)\right\rangle$

induces a non-commutative holonomy of order 10, Lemma [5.2,

31/03/02: $\langle(b, B),(d, D)\rangle$

induces a non-commutative holonomy of order 10, Lemma 5.2 .

- 48 :

33/05/01/003: CONSTRUCTION! (Theorem 7.1).

\section{REFERENCES}

[1] H. Brown, R. Bülow, J. Neubüser, H. Wondratschek and H. Zassenhaus, Crystallographic Groups of Four-Dimensional Spaces, John Wiley \& Sons, Inc., New York, 1978. MR58:4109

[2] J. Cygan, A tangential convergence for bounded harmonic functions on a rank one symmetric space, Trans. Amer. Math. Soc., 265 (1981), 405-418. MR83h:43010

[3] K. Y. Ha, J. H. Jo, S. W. Kim and J. B. Lee, Classification of free actions of finite groups on the 3-torus, Topology Appl., 121 (2002), 469-507. MR2003j:57056

[4] S. Hersonsky and F. Paulin, On the Volumes of Complex Hyperbolic Manifolds, Duke Math. J., 84 (1996), 719-737. MF97h:32036

[5] I. Kim and J. R. Parker, Geometry of quaternionic hyperbolic manifolds, Math. Proc. Cambridge Philos. Soc. 135 (2003), 291-310.

[6] K. B. Lee and F. Raymond, Topological, affine and isometric actions on flat Riemannian manifolds, J. Differential Geom., 16 (1981), 255-269. MR84k:57027

[7] K. B. Lee, J. Shin and S. Yokura, Free actions of finite abelian groups on the 3-torus, Topology Appl., 53 (1993), 153-175. MR94j:57016

[8] K. B. Lee and A. Szczepański, Maximal holonomy of almost Bieberbach groups for Heis ${ }_{5}$, Geom. Dedicata, 87 (2001), 167-180. MR.2002g:20084

[9] J. Wolf, Spaces of Constant Curvature, 5th ed., Publish or Perish, Wilmington, 1984.

[10] S. Wolfram, Mathematica, Wolfram Research, 1993.

Department of Mathematics, Sogang University, Seoul 121-742, Korea

E-mail address: kyha@sogang.ac.kr

Department of Mathematics, Sogang University, Seoul 121-742, Korea

E-mail address: jlee@sogang.ac.kr

Department of Mathematics, University of Oklahoma, Norman, Oklahoma 73019

E-mail address: kb_lee@math.ou.edu 